San Jose State University

SJSU ScholarWorks

Master's Theses

Master's Theses and Graduate Research

1992

\title{
Effects of substrate texture on the tribology of the thin film magnetic rigid disk
}

Richard Cheng-Hwa Lin

San Jose State University

Follow this and additional works at: https://scholarworks.sjsu.edu/etd_theses

\section{Recommended Citation}

Lin, Richard Cheng-Hwa, "Effects of substrate texture on the tribology of the thin film magnetic rigid disk" (1992). Master's Theses. 338.

DOI: https://doi.org/10.31979/etd.dhyw-k3vs

https://scholarworks.sjsu.edu/etd_theses/338

This Thesis is brought to you for free and open access by the Master's Theses and Graduate Research at SJSU ScholarWorks. It has been accepted for inclusion in Master's Theses by an authorized administrator of SJSU ScholarWorks. For more information, please contact scholarworks@sjsu.edu. 


\section{INFORMATION TO USERS}

This manuscript has been reproduced from the microfilm master. UMI films the text directly from the original or copy submitted. Thus, some thesis and dissertation copies are in typewriter face, while others may be from any type of computer printer.

The quality of this reproduction is dependent upon the quality of the copy submitted. Broken or indistinct print, colored or poor quality illustrations and photographs, print bleedthrough, substandard margins, and improper alignment can adversely affect reproduction.

In the unlikely event that the author did not send UMI a complete manuscript and there are missing pages, these will be noted. Also, if unauthorized copyright material had to be removed, a note will indicate the deletion.

Oversize materials (e.g., maps, drawings, charts) are reproduced by sectioning the original, beginning at the upper left-hand corner and continuing from left to right in equal sections with small overlaps. Each original is also photographed in one exposure and is included in reduced form at the back of the book.

Photographs included in the original manuscript have been reproduced xerographicaliy in this copy. Higher quality $6^{\prime \prime} \times 9^{n \prime}$ black and white photographic prints are available for any photographs or illustrations appearing in this copy for an additional charge. Contact UMI directly to order.

\section{$\mathrm{U} \cdot \mathrm{MI}$}

University Microfilms International

A Bell \& Howell Information Company

300 North Zeeb Road. Ann Arbor. MI 48106-1346 USA

$313 ; 761-4700 \quad 800: 521-0600$ 

Order Number 1348699

Effects of substrate texture on the tribology of the thin film magnetic rigid disk

Lin, Richard Cheng-Hwa, M.S.

San Jose State University, 1992

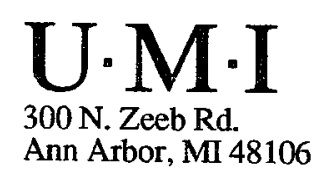




\title{
EFFECTS OF SUBSTRATE TEXTURE ON THE TRIBOLOGY OF THE THIN FILM MAGNETIC RIGID DISK
}

\author{
A Thesis \\ Presented to \\ The Faculty of the Department of Materials Engineering \\ San Jose State University \\ In Partial Fulfillment \\ of the Requirements for the Degree \\ Master of Science
}

By

Richard Cheng-Hwa Lin

May, 1992 
Approved for the Department of Materials Engineering
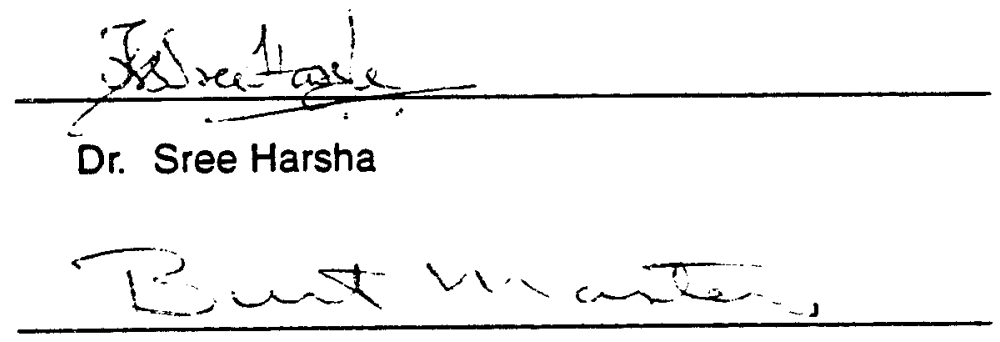

Dr. Burt Masters

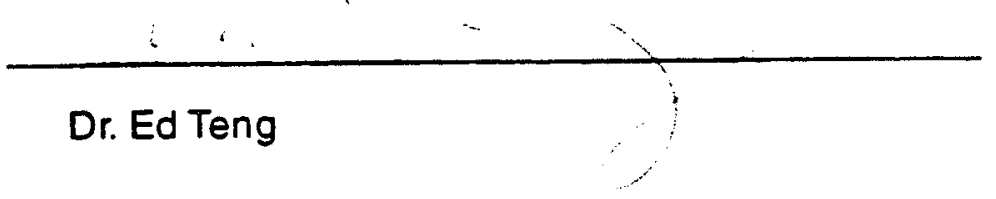

Approved for the University

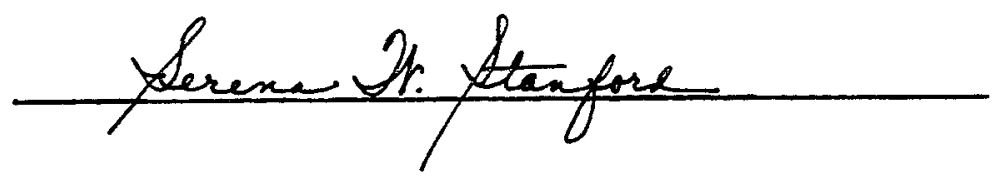




\section{ABSTRACT \\ EFFECTS OF SUBSTRATE ON THE TRIBOLOGY OF THE THIN FILM MAGNETIC RIGID DISK \\ by Richard C. Lin}

The influence of surface roughness in determining the tribological behavior of thin film magnetic rigid disks has been investigated. Electroless $\mathrm{Ni}-\mathrm{P}$ was deposited on $\mathrm{Al}$ substrates which were polished and textured circumferentially. An in-line dc magnetron sputtering system was used to deposit magnetic and carbon layers on these $65 \mathrm{~mm}$ substrates. Various texturing tapes and different texturing parameters were utilized to obtain different surface roughness values. Roughness measurements were made with Wyco and Tencor surface profilers. Measurements of stiction/friction and accelerated wear tests were studied on disks with different surface roughness. The relationships between various texturing parameters and crossing ratio are also discussed. The analysis of coefficient of friction vs. cycles of revolutions is described. On a nine legs experiment without dry buffing step, none showed any better accelerated wear test characteristics than the other. To characterize the surface texture of an area more completely, a statistical analysis software package called Statgraphics was used in this thesis. Statgraphics shows that two texturing factors, oscillation frequency and disk rotational speed are significant in changing surface morphology. 


\section{Table of Contents}

Page

I. Abstract III

II. Table of Contents IV

III. List of Figures VI

IV. List of Tables VIII

V. Acknowledgements IX

1. Introduction 1

2. Background 5

2.1. The Nature of Surfaces 5

2.2. Magnetic Recording Principle $\quad 7$

2.3. Magnetic Writing 10

2.4. Magnetic Reading 10

2.5. Principle of Dry Buff 12

2.6. Principle of Glide 13

2.7. Manufacturing Process 14

2.8. Description of Texturing Process 18

3. Analytical Instruments Employed in This Study 20

3.1. Wyko 20

3.2. Tencor 21

3.3. Lotus 24

3.4. EDC-1RPM 25 
3.5. MTS Tester

3.6. Failure Detection
26

27

28

31

57

58

66 


\section{List of Figures}

Page

1. A Schematic Drawing of the Various Layers in a Hard Disk. 2

2. Illustration of the Six Most Commonly Used Surface 4 Characterization Parameters.

3. Pictorial Display of Surface Characteristics. 6

4. Hysteresis Loop of a Hard Magnetic Film Used in a Storage 8 Medium.

5. Principles of Writing and Reading Using an Inductive Head and a 9 Longitudinal Moving Media.

6. A Typical Manufacturing Process of Thin-Film Rigid Disks. 15

7. A Typical Hard Disk Texturizer Process Set-Up. 19

8. Schematic Diagram of Wyco's 3-D Optical Interferometer. 22

9. Diagram of Tencor P-1 Long Scan Profiler. 23

10. Graphical Definition of Zero Crossing and 2-D Peaks. 37

11. High Magnification Pictures of Various Texturing Legs. 38

12. Effect of Crossing Ratio on Peak Friction (nine legs experiment). 39

13. Effect of Crossing Ratio on Range of Modulation (nine legs 43 experiment).

14. Number of Revolutions Before Failure (AWT) for the Nine Legs 44 Experiment.

15. Number of Revolutions Before Failure (AWT) for the Repeated 45 Nine Legs Experiment.

16. Effect of Crossing Ratio on Lifetime (nine legs experiment). 
17. Effect of Crossing Ratio on Lifetime (repeated nine legs experiment).

18. Effect of OSCFREQ on 3D Ra (110 RPM). 51

19. Effect of OSCFREQ on 3D Ra (300 RPM). 52

20. Effect of SSP on 3D Ra (1 HZ). 55

21. Effect of SSP on 3D Ra $(5 \mathrm{HZ})$. 56 


\section{List of Tables}

Page

1. Texturing Variables and Their Corresponding Legs. 30

2. Summary of Wyco Data on Different Textures. 32

3. Summary of Tencor Data on Different Textures. 33

4. Different Texture Processes vs. Glide Yield. 35

5. Summary of Drag Test for Different Texturing Processes. 37

6. Ra vs. Tribological Parameters for the Nine Legs Experiment. $\quad 40$

7. Texturing Parameters and Surface Responses for the Nine Legs 41 Experiment.

8. Repeat Nine Legs: Ra vs. Tribological Parameters. 46

9. Crossing Ratio vs. AWT Failure for Both Nine Legs Experiments. $\quad 47$

10. Analysis of Variance for Various Surface Responses in the Nine 53 Legs Experiment. 


\section{Acknowledgements}

I wish to express my sincere gratitude to professor Burt Masters, my thesis advisor, for his guidance and assistance throughout my thesis research and write-up. I am also grateful to Dr. Ed Teng and Professor Sree Harsha for their advice and serving on my reading committee.

My deepest appreciation goes to my grandparents. Thanks for their endless tolerance, encouragement, and support of my research study. Many thanks to my wife Jessie for her encouragement and support.

I wish to thank Bill Murphy of Nashua Corporation, Jaime Tseng, and Nelson Yeh for the loan of their Macintoshes and assistance during the thesis write-up. 


\section{INTRODUCTION}

In recent years thin film metallic disks have been manufactured extensively. They are used in high performance rigid disk drives in order to meet the requirements for high recording density. The need for lower flying heights and good contact start/stop (CSS) performance have make tribology a primary important area in the disk industry. In magnetic disk devices the slider carrying the read-write head flies within a fraction of a micron above the rigid disk over an air bearing during operation. When the unit is first started or when it is turned off, however, the air bearing is lost and the slider comes in contact with the disk. It is at this head-disk interface that the tribological problems occur. During such contact only the high points (asperities) of the disk are touching the head. A term called "true contact area" is used to describe the phenomenon. The true contact area is that portion of the apparent mating surfaces that is in intimate molecular contact. When the slider is fully loaded, the number of contacting asperities and the magnitude of the load on each asperity are related to the profiles of the mating surfaces and the material properties of the slider and the disk. In other words, the true contact area generated by a constant load depends primarily on the statistical distribution of the asperities.

These sputtered thin film disks are composed of four major layers shown in Figure 1. The base layer is an aluminum alloy substrate layer. The next layer is a nickel-phosphorus plated layer. The following layer is a $\mathrm{Cr} / \mathrm{Co}$ alloy magnetic recording layer. The next layer is a thin carbon overcoat film 
to assure good corrosion resistance. The surface layer is a lubricant protective coating layer to improve the CSS performance. The details of these layers will be explained in the background section under manufacturing process.

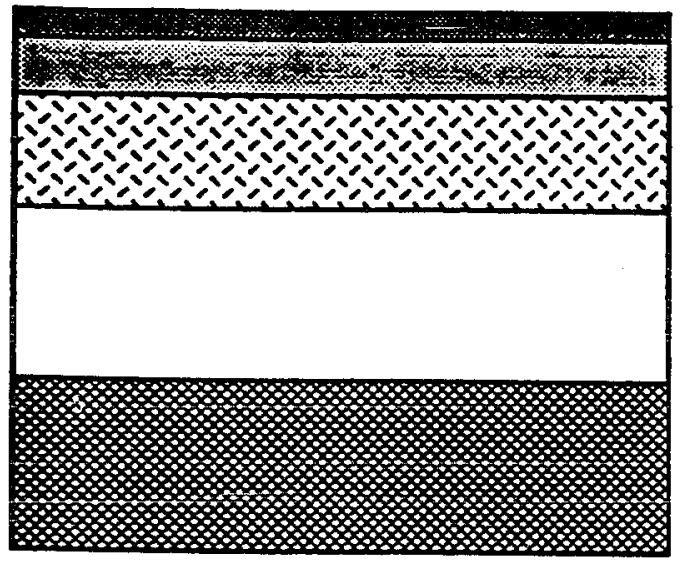

Overcoat 100-200A

Magnetic Film 200-500£

Chromium Underlayer 1000-2000A

NiP (amorphous) $10 \mu \mathrm{m}$

Al Substrate

Figure 1. A Schematic Drawing of the Various Layers in a Hard Disk.

Aluminum alloys are suitable base materials for memory disks because of their availability, cost, machineability, and low density. The nickel-phosphorus layer is used as an undercoat because it provides a hard surface for deposition of the magnetic layer. After deposition by electrolessplating, the nickel-phosphorus layer is polished to obtain a smooth mirror-like surface. Prior to sputter deposition of the magnetic recording layers, the surface is then intentionally roughened by a mechanically abrasive process known as texturing. 
There are two purposes for the texturing process. First, the texturing step is effective in reducing the contact area between head and disk, thus potentially improving the tribology (stiction, friction) characteristics. Secondly, the texturing step forms a circumferential grooved pattern which enforces the alignment of the magnetic domains along the circumferential direction. There are various texturing parameters that can describe a surface topography in terms of roughness. These include $\mathrm{Ra}, \mathrm{Rq}, \mathrm{Rp}, \mathrm{Rv}$, and $R z$. They are defined in the appendix. Some of these parameters are shown in Figure 2.

Two commonly employed criteria to describe the mechanical performance of the head-disk interface are (a) the magnitude of the stiction/friction forces that develop at the interface and (b) the durability of the protective overcoat which prevents wear of the magnetic layer. The mechanically induced geometric textures (grooves) are closely related to the tribology of the head-disk interface. In this thesis, the role of the surface roughness of thin film disks on the stiction/friction and wear properties of the head-disk interface is investigated. The statistical methods of analysis and mesurement of surface texture will be discussed. 


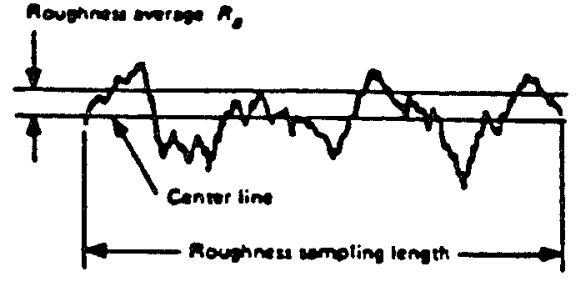

ROUGHNESS AVERAGE

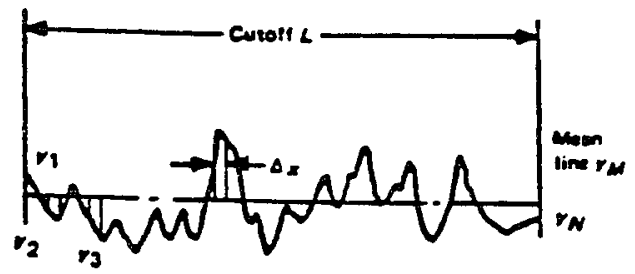

ROOT-MEAN.SQUARE (rms) ROUGHNESS $\boldsymbol{R}_{\boldsymbol{Q}}$

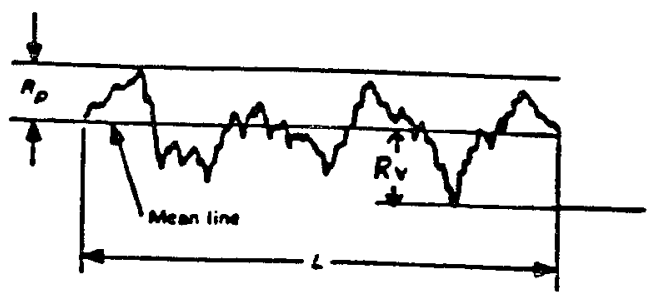

$R_{p} * R_{v}$

Figure 2. Illustration of the Most Commonly Used Surface Characterization Parameters. (35) 


\section{BACKGROUND}

\section{The Nature of Surfaces}

Solid surfaces are imperfect regardless of manufacturing methods. These imperfections can be classified with respect to orders of dimension. They range from shape deviations (three-dimensional imperfections) to surface irregularities of the order of atomic diameters. No fabrication techniques known today can produce evenly smooth surfaces on a molecular level. Even the smoothest surfaces contain irregularities with dimensions that exceed the interatomic distances. If the surfaces of contacting bodies are smooth, the contact between them is continuous within the nominal area. In practice, most engineering solid surfaces in use today are not perfectly smooth. The contact between rigid solid surfaces is discontinuous and the real area of contact is only a fraction of the nominal area. Figure 3 shows the pictorial display of surface characteristics. A key technological area where the contact between rigid bodies is of practical concern is in high density magnetic recording. In this application, the tribological properties of materials are of principal interest since magnetic recording is accomplished by the relative motion of a head over a disk surface. The principle of magnetic recording is introduced in the following section. 


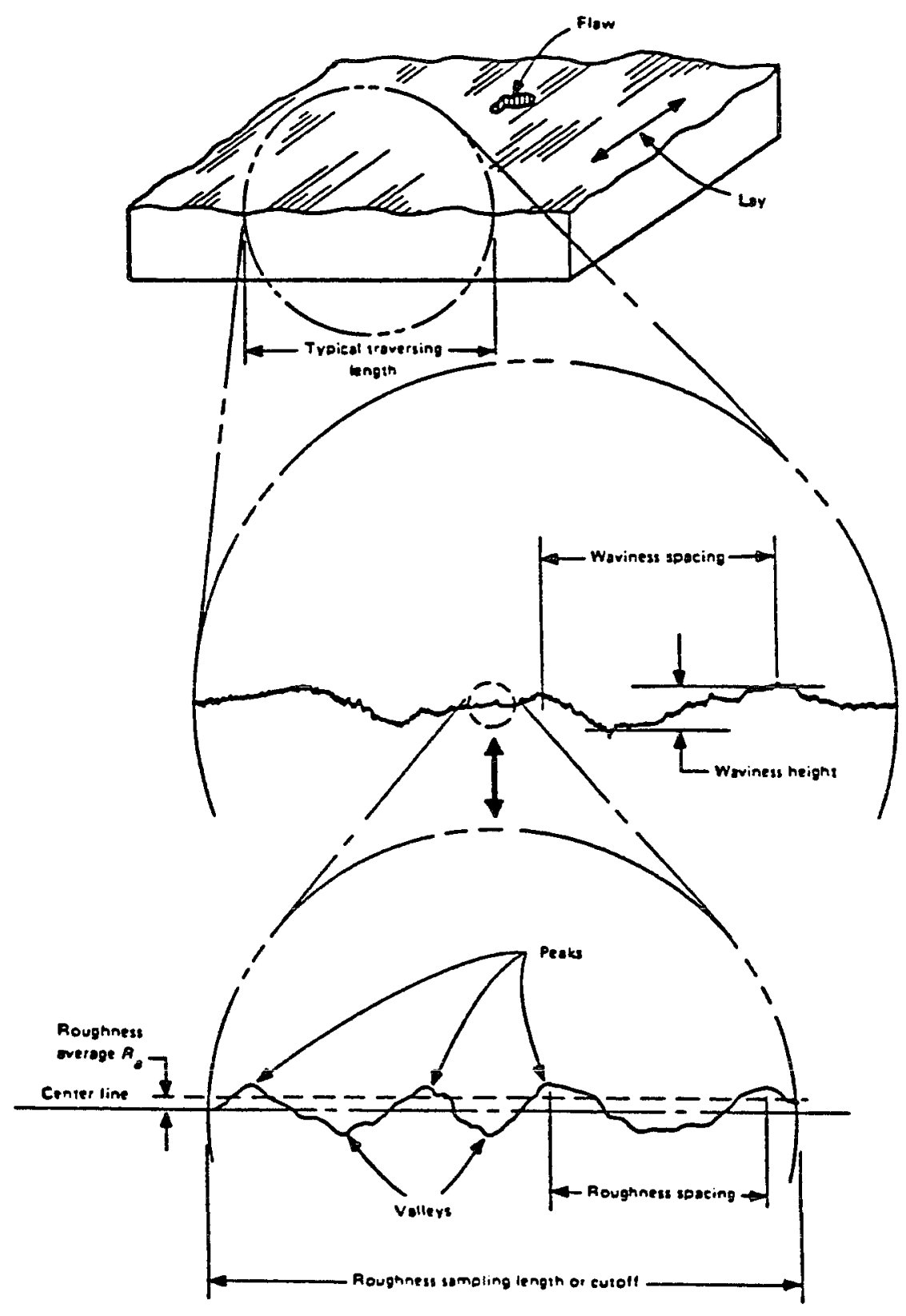

Figure 3. Pictorial Display of Surface Texture. (35) 


\section{Magnetic Recording Principle}

The magnetic recording technology is founded on magnetism and on electromagnetic induction. The magnetic properties of materials arise from the spin of electrons and the orbital motion of electrons around atomic nuclei. Since the moving charge sets up a magnetic field around it, the moving and spinning electrons act as tiny magnets. All substances exhibit magnetic effects when subjected to an external electromagnetic field. The total field is characterized by the external magnetic field strength and the magnetization. This relationship can described by a term called "hysteresis loop." Hysteresis loops can be obtained by measuring the intensity of magnetization $M$ as a function of the applied field $H$. The ratio between the magnetic field and the magnetization is commonly called magnetic permeability (u).

Magnetic recording is dependent on the phenomenon of magnetic hysteresis. Most magnetic materials exhibit this phenomenon. A typical hysteresis curve for hard magnetic material (typical for magnetic storage medium) is represented in figure 4. The ordinate designated by $M$ is the magnetization induced in the material in the presence of a field of intensity $\mathrm{H}$ (abscissa). As the strength of the field increases, domains oriented in the direction of the applied field continue to grow until the material eventually reaches saturation magnetization (Ms). Saturation magnetization is produced when all of the domains are aligned in the direction of the applied field. Any further increase in field strength provides no increase in magneti- 
zation. An important attribute of hysteresis behavior is the remanent magnetization (Mr). It is the magnetization remaining after saturation (when $H$ is reduced to zero). When the magnetic field is reversed, a critical field, called the coercive field $\mathrm{Hc}$, is required to reduce the magnetization to zero.

Figure 4 shows the locations of Ms, Mr, and $\mathrm{Hc}$.

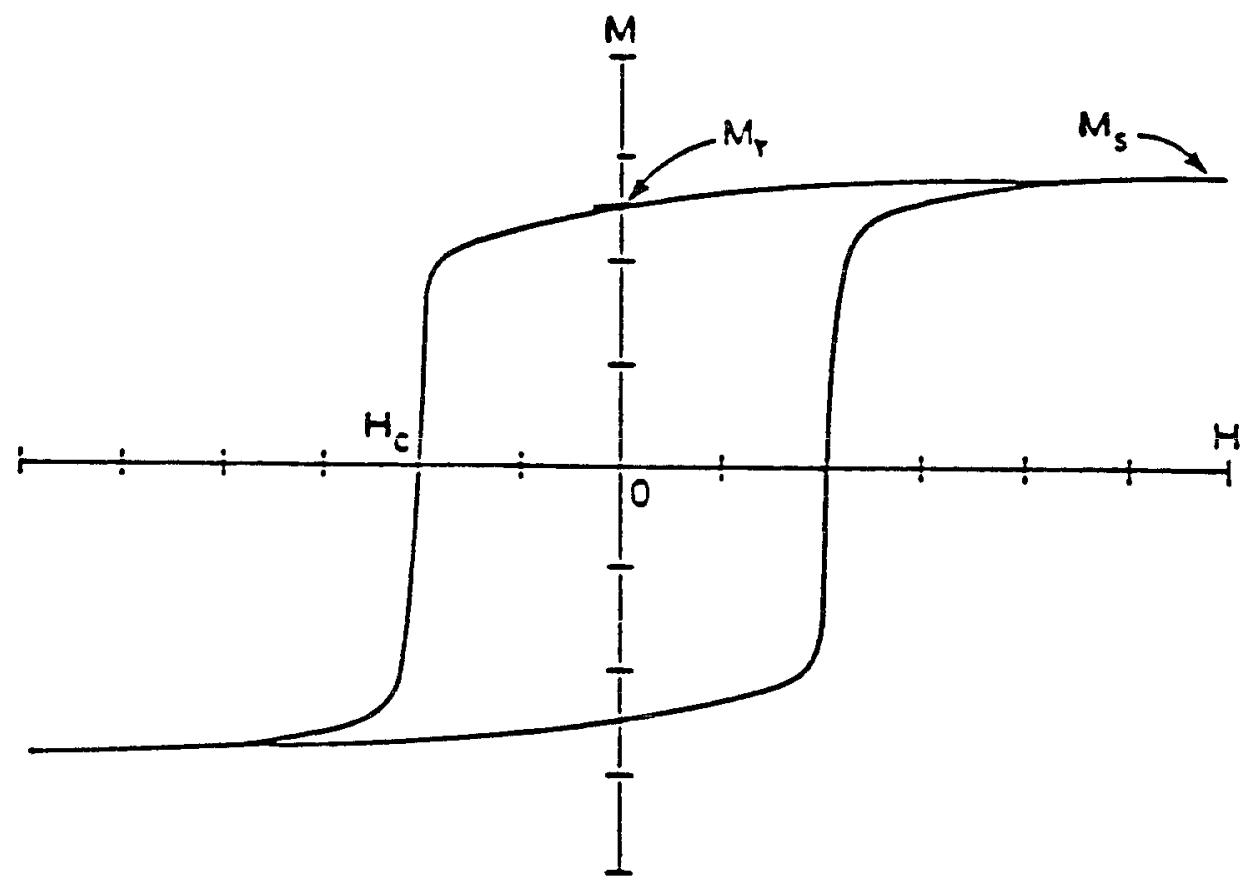

Figure 4. Hysteresis Loop of a Hard Magnetic Film Used in a Storage Medium. 
Magnetic recording is based on the interaction between a magnetic storage medium (disk) and a magnetic head (transducer), in relative motion with respect to one another. Figure 5 shows the schematic diagram of this interaction.

\section{FUNDAMENTAL PROCESSES}

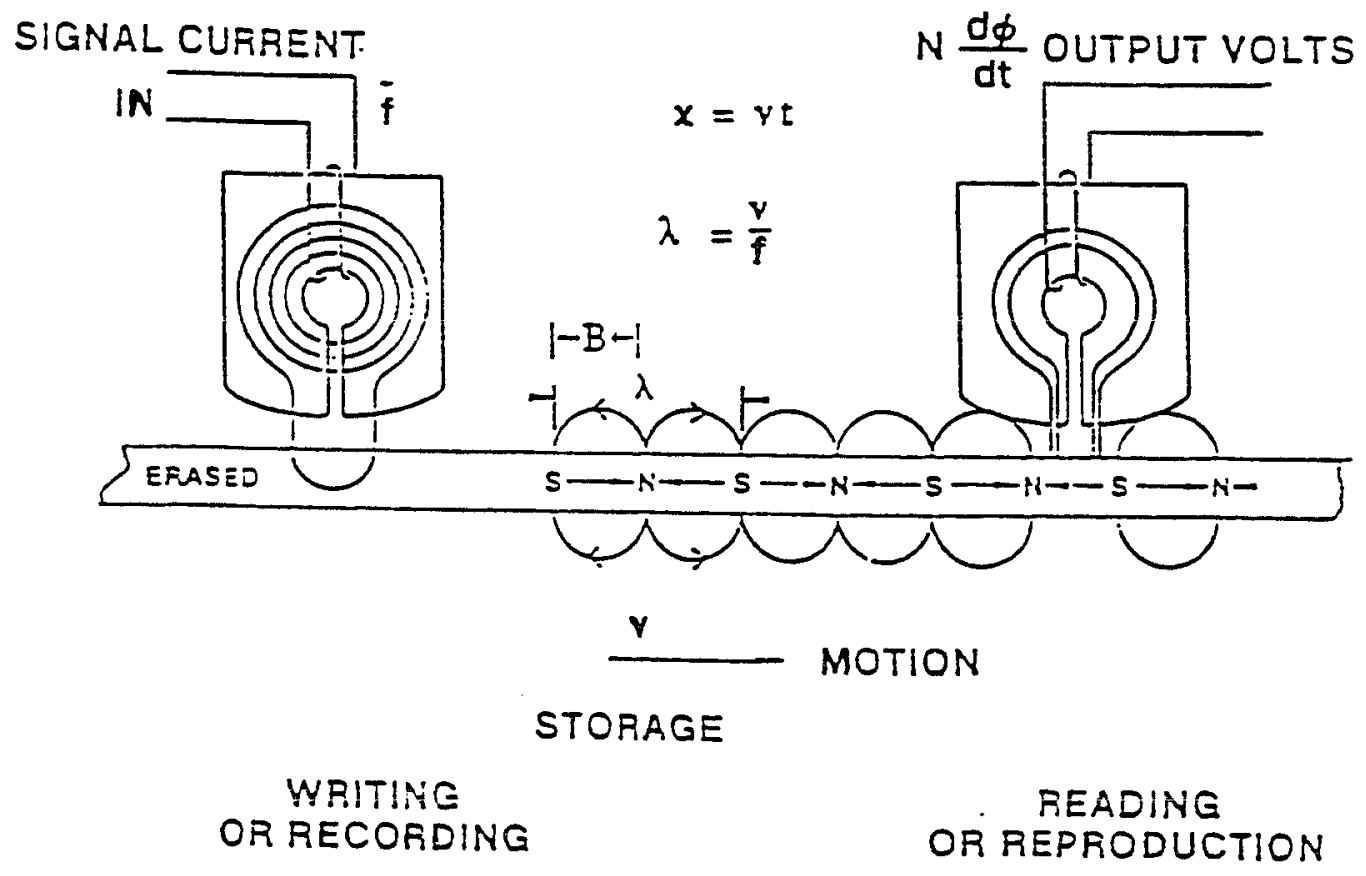

Figure 5. Principles of Writing and Reading Using an Inductive Head and a Longitudinal Moving Media. (52) 


\section{Magnetic Writing}

The recording of data in a magnetic medium is based on the induction principle. If a current flows in a coil of wire, it produces a magnetic field. The head is usually a magnetic core wound by a coil of wire. A narrow slot is cut in the core and referred to as the "gap." When electrical current is supplied, magnetic field flux lines extend from the gap region into a small region of the disk, creating a permanent magnetization pattern on the disk. This is how the data are written on the disk.

\section{Magnetic Reading}

The magnetic reading is based on the principle of induction formulated by Faraday. During reading, the recorded pattern of magnetization in the disk is coupled inductively to the reading head. As the disk spins, the region of magnetic fields of the stored data passing under the head constantly reverses its magnetization from one state to the other. This results a rapid change in induction which develops a voltage pulse. Therefore, data is read from the disk by converting flux from the disk to induced voltage provided by the head in the head winding. This voltage, after suitable amplification, reproduces the original signal. The reproduced voltage $V$ is proportional to the number of turns in the read coil.

The writing and reading of data depends on the magnetic properties of both the medium in which the data are stored and the head that writes and reads them. 
Requirements for the recording storage medium (disk) are:

i. High remanence magnetism (Mr). The voltage induced in the head during the reading process is proportional to the remanent magnetization of the recording surface.

2. Moderately large coercive force $(\mathrm{Hc})$. This is desirable to enable the magnetizing operation to proceed without accidental erasure or demagnetization.

3. The reversal of the magnetization of the medium should be accomplished over a small range of applied field strength. This helps to ensure that the states of the medium which are used for data storage will be well defined.

All these criteria are summarized by the requirement that the hysteresis loop for the magnetic medium be large and nearly square.

Requirements for the recording head are:

1. High permeability (u). Here a small flow of current through the coil around the core should yield a large magnetization (i.e., easily magnetized).

2. Low coercive force $(\mathrm{Hc})$. When the flow of current stops, the magnetization should return as nearly as possible to zero.

3. High saturation magnetization (Ms). It is needed to assure that saturation of the recording surface will occur before the head core itself saturates. In other words the transducer must provide an intense magnetic field at the surface of the medium to write the transition. The transition is defined as the region where the magnetization direction changes. It is this transition that represents one bit of data. 
4. Small Mr (remanent magnetization) of the magnetic head core. The head is not highly magnetized when not energized.

Since the recording process involves only changing the direction of magnetization in the storage medium, it is possible to erase unwanted information and record a new message in its place. This process of writingerase-rewriting can be repeated indefinitely since it is an electronic process rather than a chemical change or atomic movement. The recorded information is remarkably stable. It is almost unaffected by time, temperature, pressure, ionization, radiation or any physical changes other than the application of strong magnetic fields.

\section{Principle of Dry Buff}

The dry buff process is designed to remove the high peaks after the wet texture. This is an effective approach to achieve a better wear resistant and better glide yield surface. However, flatting of the top too much will drastically increase the real contact area and cause stiction problems. The buff process must be a controllable process in regard to the $\mathrm{Rp}$ reduction and $R p$ to Ra ratio within the Ra control range. 


\section{Principle of Glide}

Due to the never ending quest to achieve higher areal densities in thin film media manufacturing, the flying height of head/disk interface has been constantly reduced to nearly zero (contact recording). The purpose of a glide test is to detect any head/disk contacts which might degrade the performance of a disk drive.

A glide testing procedure is implemented to monitor the asperity height. The glide head used for this test is designed to have a flying height a little bit lower than the operating flying height in order to detect asperity defects or contaminants. Such testing minimizes the possibility of the head hitting disk asperities during normal operation. The glide head used for this experiment utilized a piezoelectric transducer (PZT) sensor to detect interactions. The concept is called acoustic emission. A PZT sensor is a tiny piece of piezoelectric crystal mounted on the arm of the glide head. When the glide head encounters a surface anomaly the head reacts with a sharp, violent motion which sends a vibration to the arm. The vibration excites the crystal which sends a signal to the preamp board where it is filtered and amplified. The signal is then sent to the signal processor, goes through additional gain and filter stages, and is compared with a set of DC voltages. If the signal exceeds the DC voltage then it is considered a HDI (head-diskinterference) and recorded as an encounter. Usually a man-made bump-disk with known asperity height is used to calibrate the glide tester to a specific flying height requirement. The entire disk surface is swept to determine the 
number of $\mathrm{HDI}$ defects and their locations.

Yield is one of the most important factors in regard to the profitability of a manufacturing process. The yield of glide as well as visual inspection are afiected by texture processes, especially for the disks which require low glide (i.e., less than 4 microinch flying height).

\section{Manufacturing Process}

A typical manufacturing process of thin-film rigid disks is shown in Fig. 6 in the form of block diagram. The subtrate for rigid disks is typically composed of a high quality aluminum alloy. The substrate surface is usually electroless plated with a 10 micrometers thickness of nickel-phosphorus layer. The substrate is then polished to remove most surface defects and achieve a smooth, mirror-like surface. The resulting surface has thickness of nickel about 5 micrometers. After cleaning, the substrate undergoes a mechanical texturing step utilizing a moving-tape-abrasive texturizer. Next, an in-line DC magnetron sputtering system " with double-sided deposition capabilities is used to complete the fabrication process. The Ni-P substrates are heated prior to the deposition process. The pallet upon which the disks

* A Circuit Processing Apparatus, ( located at 48603 Warm Springs Blvd., Fremont, CA. 94538) model \# V2000, was employed in the present investigation. 


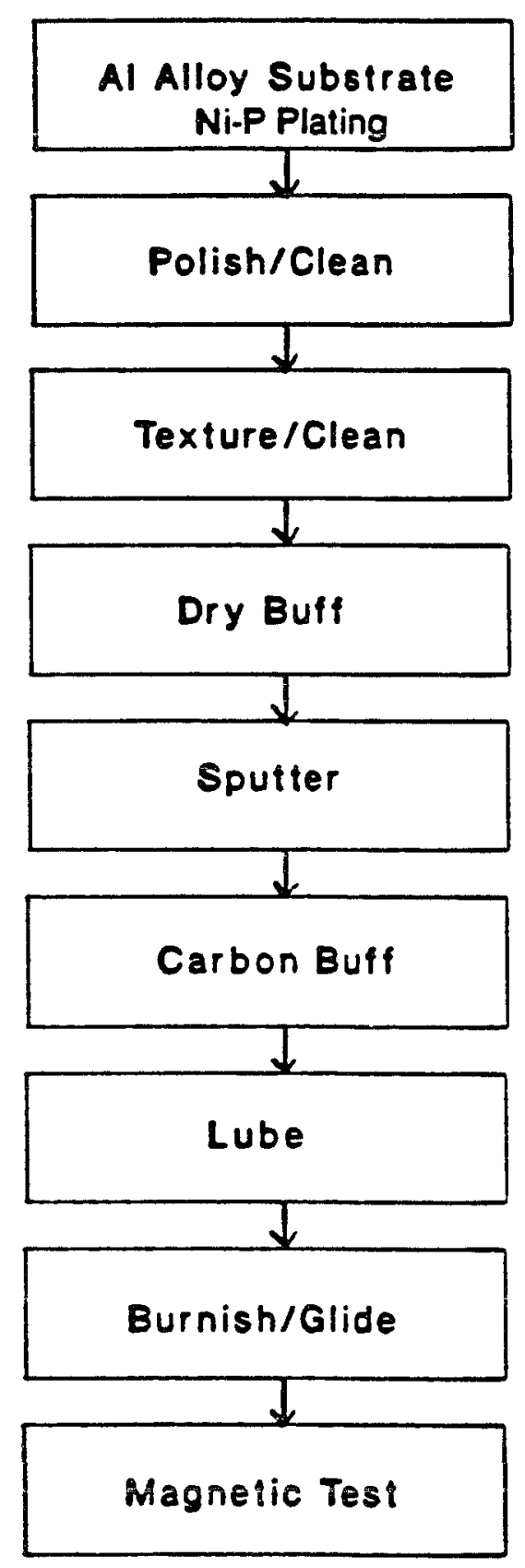

Figure 6. A Typical Manufacturing Process for Thin Film Rigid Disks. 
rests traverses at a linear speed (typically $30 \mathrm{~cm} / \mathrm{min}$ ) inside the sputtering chamber. The magnetic layer is typically composed of $1000 \mathrm{~A}$ of chrome underlayer and $500 \mathrm{~A}$ of $\mathrm{Co} / \mathrm{Cr}$ layer. The carbon layer, with typical thickness of $200 \mathrm{~A}$, is deposited on top of the magnetic layer. The objective of carbon overcoat is to provide wear and corrosion resistant protection for the metal underlayers. The disk surfaces are then carbon buffed ${ }^{\#}$ to smoothen the carbon surface. A dip lube step is applied next to obtain about a 25 A thick lubricant layer. The lubricant is a dilute solution of Freon TF and Fluorinet mixture. In the dip lube process, the disks are lowered into the lubricant bath and withdrawn at a constant prescribed rate. The lubricated disks are burnished and glide height tested for defects. In the burnishing process, a titanium carbide head is used to break off projecting asperities and to remove the dust particles on the disk surface. The disks then undergo various durability tests such as accelerated wear test (AWT) and CSS tests. The purpose of AWT is to determine the relative durability of the disk in a short test period (on the order of minutes or hours). In the AWTs, the head and the disk surfaces are slid in contact with no air bearing present to accelerate the interface wear. The CSS test is another relative measurement of the life of a rigid disk drive. In this test, the spindle is programmed to accelerate to full speed, from rest, stay at full speed for a short time, then decelerate to zero. It then stops for a short period of time. This simulation of drive acceleration and deceleration is necessary because they measure wear during start/stop

\# An Exclusive Design Company, (914 S. Claremont St., San Mateo, CA. 94402), model \# 872, was used in the present investigation. 
cycles and the resulting friction. A whole start/stop cycle lasts for about sixty seconds. After passing the prescribed durability tests, the disks are magnetically tested. This step is to see if all parameters are within the specifications. These parameters include: amplitude, resolution, bit shift, overwrite, and pulse width, etc. Finally, the good disks are ready to ship to prospective customers. 


\title{
Description of Texturing Process
}

\begin{abstract}
A Hard Disk Finisher (HDF) texturizer is usually used to accomplish the texturing step. Most texturizing models use a dual station tape carrier system. Different sizes of alumina abrasive tapes and cotton clean tape, both supported by polyurethane rollers, clamp the rotating disk surface in a vertical direction as shown in fig. 7. The vertical component of movement during texturing, where the abrasive grid is actually touching the surface, is called oscillation amplitude. Oscillation frequency is the cycles per second such movement occurs in the vertical direction. The function of the clean tape is to remove nickel debris formed during texturing. Both texturing and cleaning tapes are continuously advanced along with the worn tapes.
\end{abstract}

In the texturing process, it is difficult to get a highly reproducible and uniform surface because of the formation of both asperities or bumps due to hard nickel nodules as well as to cold-welded Ni particles created during texturing. A consistent texturing process is needed to keep the stiction/friction under control. But because of the above mentioned reasons, it is hard to maintain such a process.

* In the present study, an EDC 872 model was used. This model has a new high torque, low speed spindle motor and is capable of high crossover angle texturing applications due to its superior torque, speed range, and control. 


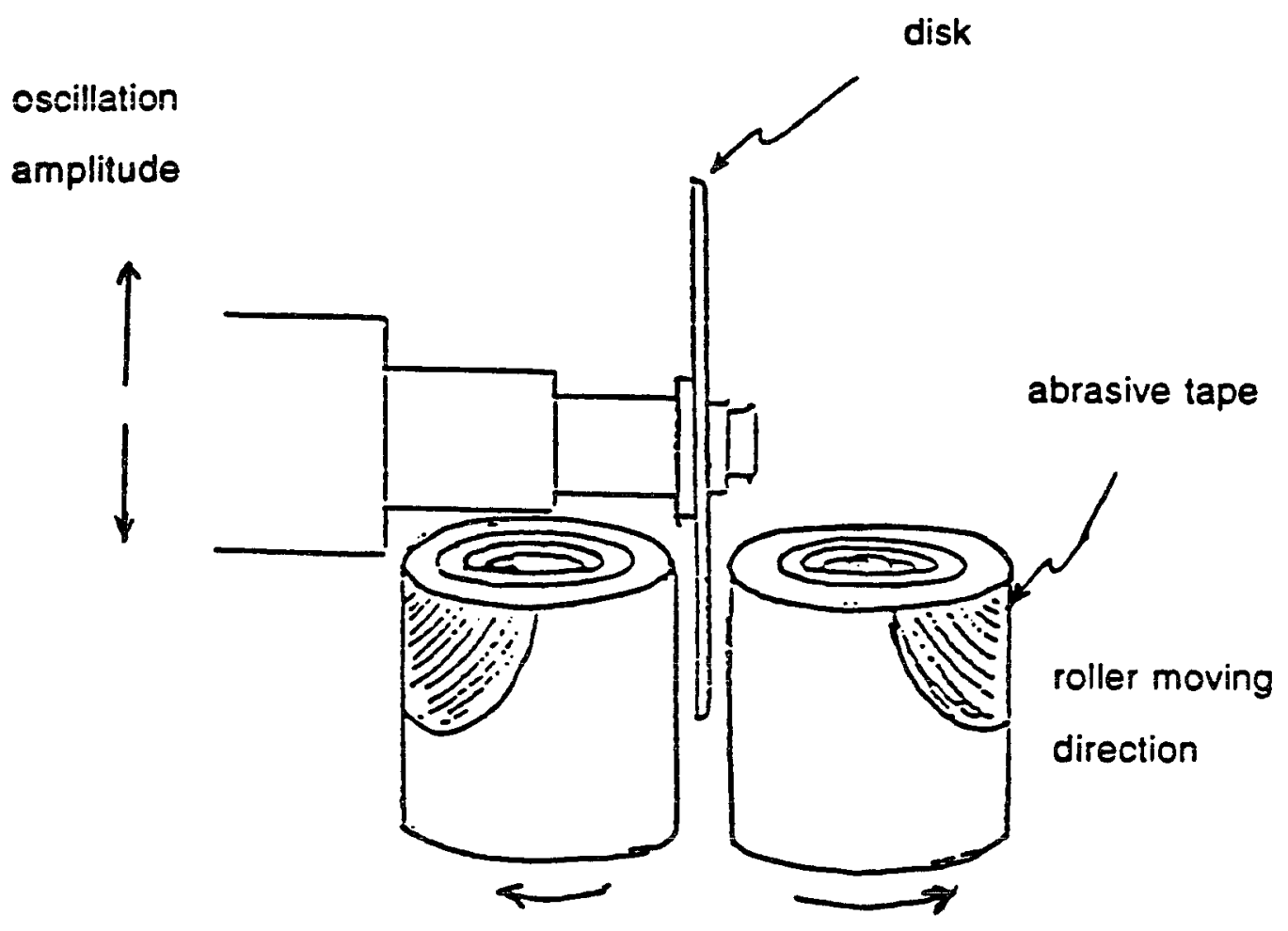

Figure 7. A Typical Hard-Disk Texturizer Process Set-up. 


\section{ANALYTICAL INSTRUMENTS EMPLOYED IN THIS STUDY}

\section{Wyko^.}

Wyko Corporation's TOPO-3D non-contact surface profiler is operated on the principle of direct phase-detecting interferometry. Interferometry offers exemplary resolution and involves no physical contact with the surface to be studied. Optical interferometry offers significant advantages for studying surface texture. The only probing tool used is a low-intensity beam of light, and so the process is nondestructive. In principle, interferometry can resolve surface irregularities in the order of Angstroms. Moreover, the technique offers valid study of the surface, since the area viewed is large in proportion to the size of individual surface features.

The principle of two beam optical interferometry occurs when two light waves are brought together to interact. A half-silvered mirror called a beam splitter divides an incident light beam into iwo parts. One beam refilects off a flat reference mirror; the other beam bounces off the surface of the specimen being studied. The two beams rejoin at the beam splitter. When the beams recombine, one part of the second beam will be in phase with the first (bright region), other parts may be out of phase (dark regions). The pattern of brightdark-bright cycle is repeated across the interference image. The pattern is known as a fringe pattern.

^ Wyko Corporation 3-D Topo, 2650 E. Elvira Road, Tuscon, Arizona 85706. 
Bumps and dips on the surface of the specimen change the path length traveled by the first beam, alter the spatial relation between the two beams and the shape of the fringes. The interference images have various brightnesses at each point, which are analyzed to reveal the wave phase. This information corresponds to surface height for the points on the sample. Thus, it magnifies the surface texture configuration.

The reference mirror in the interferometer is mounted on a piezoelectric transducer (PZT). During measurements, a computergenerated voltage can be applied to the PZT to control its motion. This allows the use of electronic-phase-detecting techniques to measure the phase of the interference pattern. In addition, the computer provides the software program that further analyzes the surface height data and displays the result in two- and three- dimensional color diagrams. Figure 8 shows the schematic diagram of Wyko's TOPO 3-D interferometer.

\section{Tencor ${ }^{@}$.}

The Tencor P-1 Long Scan Profiler is a computerized, highly sensitivity surface profiler for measuring roughness, waviness, and step height in a variety of applications. It features the ability to measure micro-roughness with 1 angstrom resolution over short distances. Figure 9 is a view of the Tencor P-1 Long Scan Profiler.

(a) Tencor Instruments model P-1, 2400 Charleston Road, Mountain View, CA. 94043. 


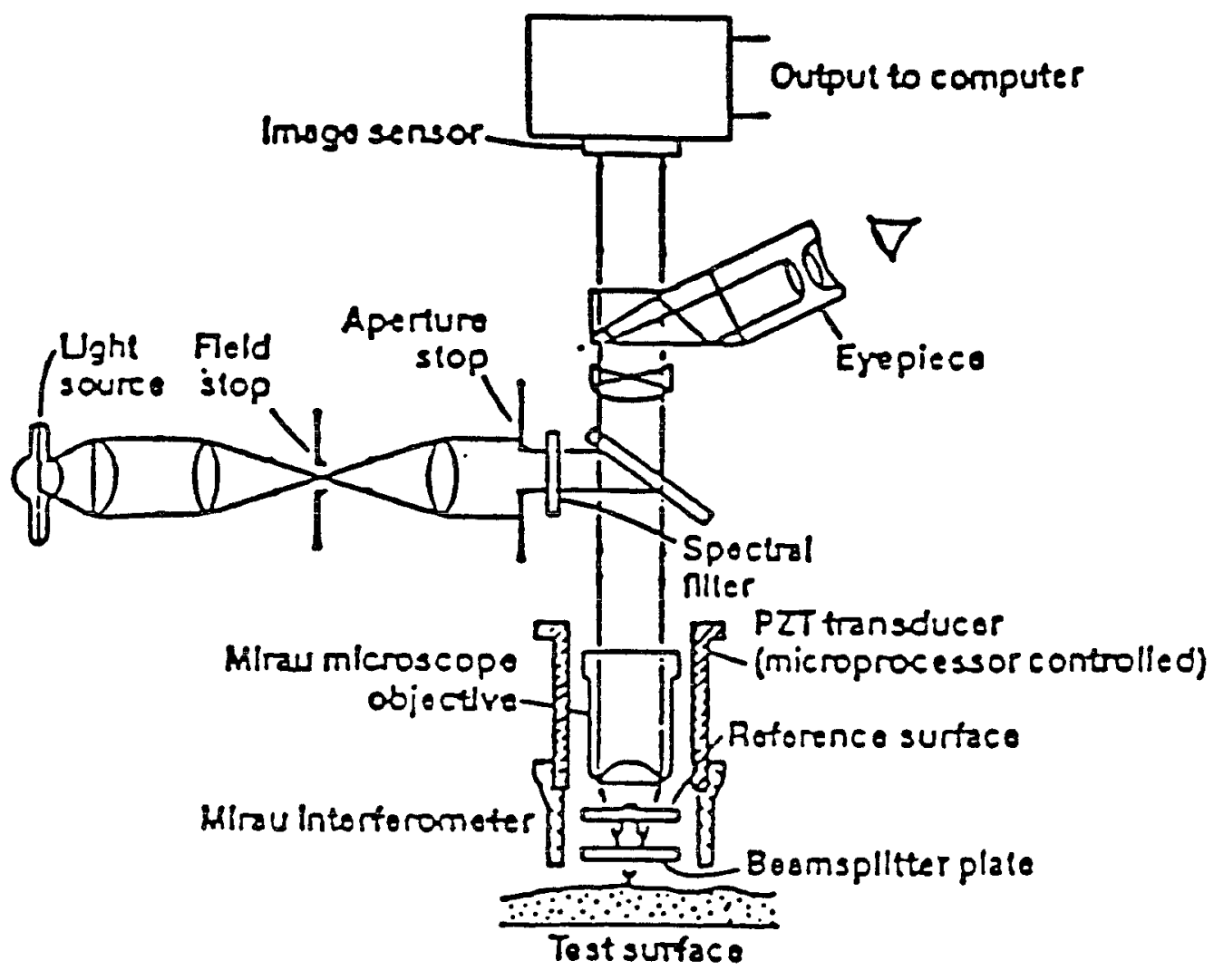

Figure 8. Schematic Diagram of Wyko's 3-D Optical Interferometer. (Wyko Corporation) 


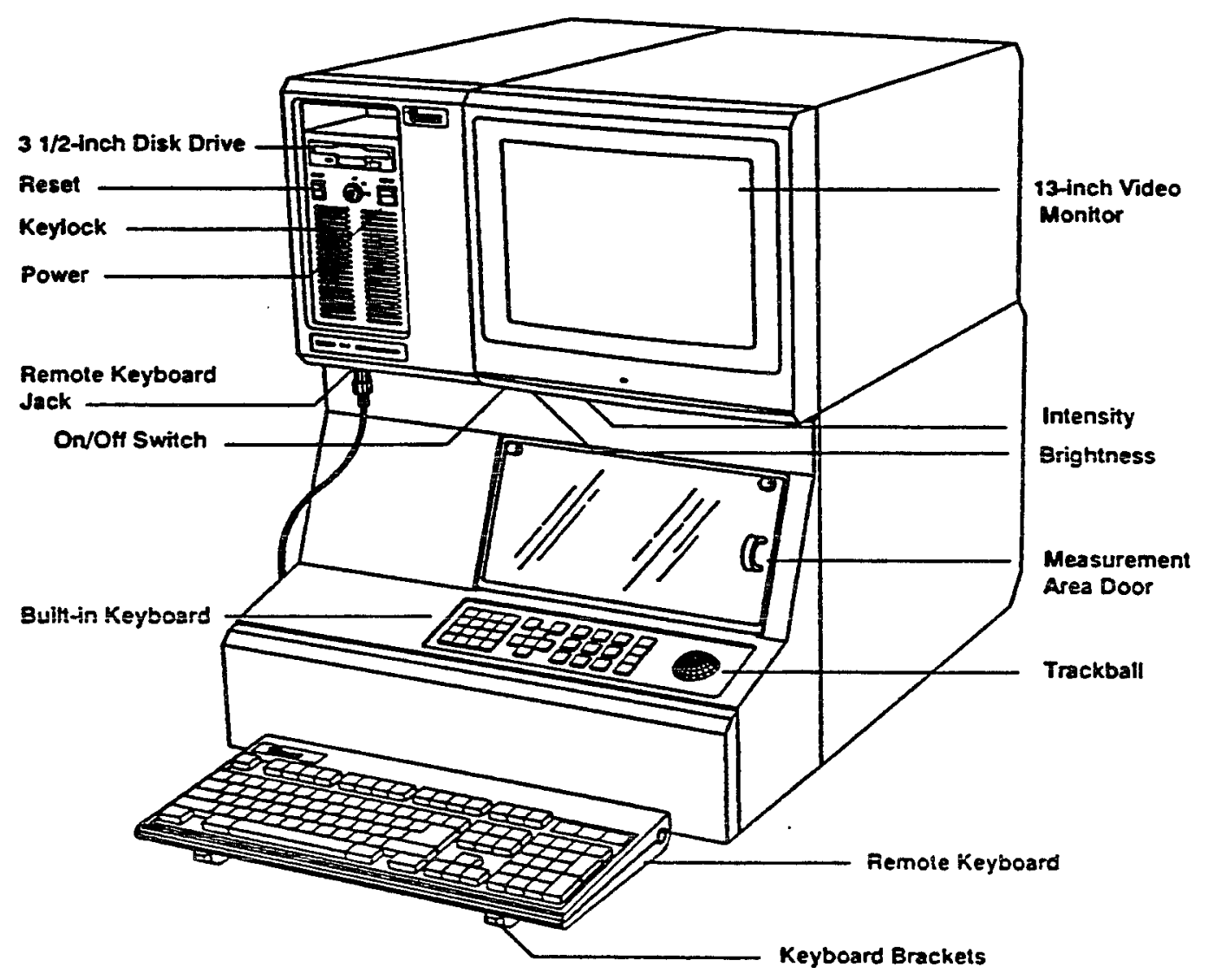

Figure 9. Tencor P.1 Long Scan Profiler. (Tencor Corporation) 
The instrument is mechnically oriented. It involves dragging a stylus across a testing surface at a constant velocity. The rising and dipping of the stylus records the terrain of the specimen's surface over a specific distance. An electronic amplifier is used to boost the signal from the stylus, which is recorded by a chart recorder. The electrical signal from the pickup mechanism represents the relative movement between the stylus and the specimen. The signal output is fed through a suitable analog-to-digital conversion and fed to a digital computer. The Tencor P-1 has a built-in PC/AT controller with 20 Mbytes of disk storage. It is capable of providing up to 6000 data points per profile.

For the signal to be truly representative of the surface profile, the stylus must traverse a path parallel to the general shape of the surface. The stylus has various radii of curvature ranging from 2 to 10 microns. During measurements the stylus tip exerts great pressure on the specimen's surface, which can compress and alter its characteristics. In contrast to optical interferometry, the stylus profilometry is destructive. Therefore, noncontact type instruments are generally preferred.

\section{Lotus\$ -}

Lotus Technologies' Model 5020, commonly referred to as "StictionFriction Event Analyzer" (SFEA), is a rigid single disk spin stand. This unit

\$ Lotus model \# 5020, 419 Lano Ct., San Jose, CA., 95128. 
is capable of acquiring static and kinetic friction forces on a single-disk level by measuring the tangential force at the head-disk interface. A manual chuck, carriage, and head load mechanism are provided to mount the disk, position the disk, and load the head at the desired disk radius. Two semiconductor strain gauges are attached to a flexible head supported arm. This arm supplies a voltage output proportional to the strain that is imposed on the head. Strain gauge output is then processed through an analog-to digital converter and is displayed on a storage oscilloscope.

\section{EDC-1RPM+}

EDC's $1 \mathrm{rpm}$ tester consists of: a spindle motor that has constant torque at $1 \mathrm{rpm}$, a gram load block of 4.5 grams, a semiconductor strain gauge with frequency response of $300 \mathrm{HZ}$, and a chart recorder. These are coupled to the head slider to sense and record the friction characteristics of the head disk combination.

A disk is attached to a spindle motor with a clamp which secures its position. A read/write head-slider is pressed down to a disk with a vertical micro-stage at a $4.5 \mathrm{gram}$ load of force. The head is originally rested on the surface of the disk to be tested. The motor is then started with a controlled torque and the disk is rotated from the initial static position. The friction trace is then plotted on the recorder for one complete revolution of the disk. As the

+ Exclusive Design Company model \# 1rpm tester, 914 S. Claremont St., San Mateo, CA 94402. 
time progresses, the head is left to continuously drag over the disk's surface for a long period of time. The friction is recorded every hour. This test is to supplement the standard test of 15,000 start-stop cycles and simulate the wear test results. Another common name for this test is called drag test.

\section{MTS Tester! -}

The MTS burnish/glide unit is a single spindle disk tester designed for the precision glide test in the computer memory industry. It can perform burnish and glide testing simultaneously. The MTS glide capability is designed to detect the asperities on the media surface before performing certification.

Microscopic roughness on a media surface forces air streams flowing over the disk into currents which can modify the airfoil dynamics and cause heads to vibrate. Since read/write amplitudes are a function of head flying height (heads flying further away from disk will produce smaller voltage), variations in flying height can degrade performance. Thus, the flying height should be low enough to give strong magnetic coupling yet high enough to prevent head crash and wear. The glide test involves flying a glide sensor over the disk in order to detect HDis. The principle of glide has been introduced in the background section.

! MTS Tester Burnish/Glide Unit, 2091 Fortune Dr., San Jose, CA., 95131. 


\title{
Failure Detection
}

\begin{abstract}
A variety of methods and failure criteria have been used in accelerated wear testing (AWT). For single component testing level, visual detection of the wear tracks have been the common practice. The other common test involves using a strain gauge amplifier. When a wear track is formed, the amplifier will pick up a signal and produce a fluctuating trace in plotter. Other methods include measuring temperature at the slider surface (8), detecting debris with piezoelectric transducer equipped sliders (9), or measuring conductivity between slider and disk (10). The above mentioned three methods required specially prepared sliders that are not readily available. Furthermore, Gataen et al. (11) used a particle counter to detect wear particles generated during sliding contact; T. Kita et al. (12) first reported the use of acoustic emission transducers to detect contact between slider and disk. In this study, both the visual detection method and strain gauge amplification method were utilized.
\end{abstract}




\section{EXPERIMENTAL}

There were two major experiments designed for this thesis. The first one was the comparison of various types of texturing processes where three different abrasive tapes were evaluated. The abrasive tapes are made of aluminum oxide particles. The three types are 3 um STEY, 2 um STEY, and 2 um STPY. The symbol $S$ stands for special tighter particle distribution, the $T$ stands for block surface, the $E$ stands for hard binder, the $P$ stands for soft binder, and the $Y$ stands for normal curing process. The difference between STEY and STPY is that the STEY uses hard chemical binding while STPY uses soft chemical binding. The nomenclature of the texturing processes are as follows: Process I - dry buffed with 3 um STEY abrasive tape; Process II - no buff with 2um STPY abrasive tape; Process III - dry buffed with 2 um STEY abrasive tape; Process IV - no buff with 2 um STEY abrasive tape. Characterizations involved surface roughness measurements, glide yield, stiction/friction, and wear testing.

Surface roughness was characterized by $R a, R p, R q$, and $R z$. The equipment used in the measurement were the Tencor P-1 and 3-D Wyko units. Glide yield data were taken by the MTS burnish/glide unit which was calibrated at $5 \mathrm{u}$ " flying height. Stiction/friction values were taken from the standard drag test from the Certus unit and wear data was taken from the standard AWT procedure by the Lotus unit.

The second experimental design consisted of nine legs. The objective 
for this experiment was to design various texturing surfaces with different crossing ratios and obtain their tribological responses. Each leg consisted of 14 disks. All the disks used were $65 \mathrm{~mm}(3.5 ")$ in diameter. These disks were textured by the EDC-872 texturizer with STEY abrasive tape and sputtered by a D-C magnetron in-line sputtering system (CPA). The texturing variables were oscillation amplitude, oscillation frequency, and disk rotational speed (SSP). Table 1 shows these variables and their corresponding legs. The design format used for this experiment was $2 \times 2 \times 2$ or $2^{3}$ factorial design. Here the two extreme levels of each of the three variables were used to illustrate what main effects and interactions were significant. The two levels for SSP were 110 and 300 RPM. For oscillation amplitude the two levels were 0.05 and 0.2 inches. The two levels for oscillation frequency were 1 and $5 \mathrm{HZ}$. This $2^{3}$ factorial design resulted in several different texturing surfaces with various roughness. Both the Tencor (with 2.5 um radius stylus) and Wyco (refer to analytical instruments) surface profilometers were used to characterize the surfaces. The tribological reponses of the 9 legs texturing experiments were also recorded. These included the standard drag test from the Certus unit and the accelerated wear test (AWT) from the Lotus unit. AWT were performed at $735 \mathrm{rpm}$ at the radius of $0.65 "$. Most of the experiments involved continuous drag testing at a fixed speed. However, at regular intervals during the test, the disk was rotated at a slow speed (1rpm) and the frictional force during one revolution was measured and averaged to give the average friction per revolution. This test is called EDC-1RPM test. 
TABLE 1. TBXTURING VARIABLEB AND THEIR CORREBPONDIMG IEGS

$\begin{array}{cccc}\text { LEG } & \begin{array}{c}\text { OSC } \\ \text { AMP }\end{array} & \begin{array}{c}\text { OSC } \\ \text { FREQ }\end{array} & \begin{array}{c}\text { SSP } \\ \text { (INCH) } \\ \text { (HZ) } \\ \text { (RPM) }\end{array} \\ 1 & 0.20 & 5.0 & 300 \\ 2 & 0.20 & 5.0 & 110 \\ 3 & 0.20 & 1.0 & 300 \\ 4 & 0.20 & 1.0 & 110 \\ 5 & 0.05 & 5.0 & 300 \\ 6 & 0.05 & 5.0 & 110 \\ 7 & 0.05 & 1.0 & 300 \\ 8 & 0.05 & 1.0 & 110 \\ 9 & 0.10 & 2.5 & 205\end{array}$




\section{RESULTS AND DISCUSSION}

The average of surface roughness parameters of the first experiment is given in Table 2 (Wyko data) and Table 3 (Tencor data). These parameters were measured at four different stages during processing (refer to Fig. 6). These stages are: after we'stexture, after dry buff, after sputter coating, and after carbon buff. Each data point in Table 2 and Table 3 is the average of 6 raw data. The ratios between $R p$ to $R a$ are included both in Table 2 and Table 3 for comparison between different texture processes. One can see from both tables that the Process I texture has the highest average surface roughness ( $\mathrm{Ra}$ ) followed by Process III texture. The Process II had the lowest average surface roughness. This is not a surprise since 3 um abrasive tape has larger size of grains than 2 um tape. These larger grains formed deeper grooves during texturing and produced rougher surfaces.

The STEY tape is much more aggressive than the STPY tape. The STEY based texture processes I, III, and IV produce higher Ra and Rp values (see Table 2) than does the STPY process II. Table 3 shows a large reduction of $R p$ by the dry buff process while Table 2 does not. The Rp to Ra ratio measured by Wyko and Tencor also seems different. No clear trend is indicated by Wyko data, while the Tencor data shows a more consistent trend. This result seems to indicate that the Tencor instrument is a better instrument for texturing process control. 
TABLE 2. BUMGARY OF WYCO DATA ON DIFFERENT TEXTURES

$\begin{array}{lrrrr}\text { GROUP I } & R a(A) & R q(A) & R p(A) & R p / R a \\ \text { PROCESS I: } & & & & \\ \text { AFTER TEXTURE } & 81.9 & 103.2 & 314.0 & 3.83 \\ \text { AFTER DRY BUFF } & 75.9 & 94.8 & 312.0 & 4.11 \\ \text { AFTER COATING } & 88.8 & 109.3 & 304.0 & 3.42 \\ \text { AFTER CARBON BUFF } & 90.2 & 113.7 & 468.0 & 5.19 \\ \quad \text { AVG } & 84.2 & 105.3 & 350.0 & 4.14 \\ \quad \text { STD } & 6.6 & \mathbf{8 . 2} & 79.1 & 0.75\end{array}$

PROCESS II:

AFTER TEXTURE

AFTER COATING

31.1

39.1

132.0

4.24

33.2

42.1

179.0

5.39

AFTER CARBON BUFF

44.7

256.0

7.21

AVG

33.3

42.0

189.0

5.60

STD

2.2

2.8

63.0

1.50

PROCESS III:

AFTER TEXTURE

AFTER DRY BUFF

50.7

63.6

208.0

4.10

47.8

59.9

185.0

3.87

AFTER COATING

57.7

73.2

206.0

3.57

AFTER CARBON BUFF

63.1

78.7

247.0

3.91

54.8

68.9

212.0

3.86

STD

6.9

8.6

26.0

0.20

PROCESS IV:

AFTER TEXTURE

AFTER COATING

43.1

54.2

174.0

4.04

53.0

67.1

237.0

4.47

AFTER CARBON BUFF

48.0

60.4

213.0

4.44

AVG

60.6

208.0

4.32

STD

48.0

6.5

32.0

0.24 
TABLE 3. BUMALAY OF TENCOR DATA ON DIFFERENT TEXTURES

$\begin{array}{lrrrrr}\text { GROUP ID } & R a(A) & R g(A) & R p(A) & R z(A) & R p / R a \\ \text { PROCESS I : } & & & & & \\ \text { AFTER TEXTURE } & 81.8 & 117.6 & 1064.0 & 862.5 & 13.00 \\ \text { AFTER DRY BUFF } & 63.8 & 79.0 & 280.0 & 472.8 & 4.39 \\ \text { AFTER COAT } & 61.2 & 75.8 & 235.5 & 441.5 & 3.85 \\ \text { AFTER CARBON BUFF } & 60.8 & 77.8 & 290.1 & 466.0 & 4.77 \\ \quad \text { AVG } & 66.9 & 87.6 & 467.4 & 560.7 & 6.50 \\ \quad \text { STD } & 10.0 & 20.1 & 398.4 & 201.6 & 4.30\end{array}$

PROCESS II:

$\begin{array}{llllll}\text { AFTER TEXTURE } & 30.5 & 47.2 & 488.0 & 388.8 & 16.00\end{array}$

$\begin{array}{lllll}30.7 & 44.8 & 448.7 & 346.5 & 14.60\end{array}$

AFTER CARBON BUFF $25.7 \quad 32.5 \quad 137.9 \quad 209.5 \quad 5.37$

$\begin{array}{llllll}\text { AVG } & 29.0 & 41.5 & 358.2 & 315.7 & 12.00\end{array}$

$\begin{array}{llllll}\text { STD } & 2.8 & 7.9 & 191.8 & 93.4 & 5.80\end{array}$

PROCESS III:

AFTER TEXTURE

AFTER DRY BUFF

53.0

68.5

$304.3 \quad 430.6$

5.74

AFTER COAT

43.5

53.5

160.6

305.0

3.69

AFTER CARBON BUFF

43.5

56.0

210.7

350.2

4.84

AVG

41.5

52.2

173.0

309.1

4.17

STD

45.4

57.6

$212.2 \quad 348.7$

4.61

5.2

7.5

$65.0 \quad 58.3$

0.89

PROCESS IV:

AFTER TEXTUR

AFTER COAT

40.7

$\begin{array}{lll}51.8 & 270.5 & 325.6\end{array}$

6.65

AFTER CARBON BUFF

46.8

59.3

\section{5}

378.8

6.16

41.8

52.5

$152.5 \quad 295.5$

3.65

$\begin{array}{llll}54.6 & 237.1 & 333.3 & 5.48\end{array}$

STD

43.1

$3.4 \quad 60.3 \quad 34.4$

1.32 
Table 4 shows the result of texture vs. glide yield for different texturing processes. It can be seen from table 4 that process III's (2um STEY with dry buff) $94.7 \%$ yield is much higher than process IV's (2um STEY without dry buff) $47.4 \%$. The Process IV disks had poor glide yield and the reason for that is the surface of these disks contained cold welded spots. The cold welding of cutting debris is caused by the friction between abrasive tape and disk surface in a typical texture process. Since the Process IV disks were not dry buffed, those spots could not be removed as they were for Process I and III disks. Table 4 also suggests that process I's ( 3 um STEY with dry buff) $100 \%$ yield is higher than process III's ( 2 um STEY with dry buff) $94.7 \%$. This indicates that 3 um tape is relatively better than 2 um tape with respect to glide yield. Process II (2 um STPY without dry buff) had a unusually high glide yield - $93.8 \%$ even without the aid of dry buffing step. The reason is probably that STPY is a much better abrasive texturing tape than STEY. The STPY presumably produces little or no texturing debris which translates to high glide yield. The relatively low $R a$ and $R p$ values produced by STPY tape are a good indication of surface cleanliness and uniformity.

The drag test results for the texturing processes are given in Table 5. Three friction coefficients are tabulated in this table. They are as follows: static friction coefficient (Usf.), kinetic friction coefficient (Ukf.), and maximum kinetic friction coefficient (Umax.). The results demonstrate that there isn't much difference in kinetic friction coefficient values (Ukf) among the different processes. It seems the Uave parameter is not a sensitive character to correlate with the processes. 


\begin{tabular}{|c|c|c|c|c|}
\hline & TOTAL & & GLIDE & AVERAGE \\
\hline GROUP ID & PÁSS & FAIL & YIELD $(\&)$ & $\operatorname{Rp}(A)$ \\
\hline $\begin{array}{l}\text { PROCESS I. } \\
\text { ( } 3 \text { UM STEY Dry Buffed) }\end{array}$ & 22 & 0 & 100.0 & 290.1 \\
\hline $\begin{array}{l}\text { PROCESS II. } \\
\text { ( } 2 \text { Um STPY No Buffed) }\end{array}$ & 15 & 1 & 93.8 & 137.9 \\
\hline $\begin{array}{l}\text { PROCESS III. } \\
\text { (2um STEY DrY Buffed) }\end{array}$ & 18 & 1 & 94.7 & 173.0 \\
\hline $\begin{array}{l}\text { PROCESS IV. } \\
\text { (2um STEY No Buffed) }\end{array}$ & 9 & 10 & 47.4 & 152.5 \\
\hline
\end{tabular}


The 9 legs texturing experiments produced different cross angle surfaces which can be characterized by $Y$ over $X$ zero crossing ratio. Figure 10 shows the graphical definition of zero crossing and 2-D peaks. The $X$ zero crossing is defined as the average of the distance between the zero crossing found in the $X$ direction while $Y$ zero crossing is the average of the distance between the zero crossing in the $Y$ direction. Leg \#2 has the highest crossing ratio. This can be seen in a high magnification (200x) picture in Figure 11. Fig. 11 also shows the high magnification pictures for various experimental legs. Leg $\# 1$ has the second highest crossing ratio. It is understood that as the crossing ratio increases the contact area between the head and the disk decreases. Since the contact is only at the high points on the disk surface, the lesser the contact the lesser the friction force. As may be seen in Table 6, the EDC 1 RPM íriction test results in the lowest peak friction $(1.12 \mathrm{gm}$ ) for leg \#2 which confirms the hypothesis. Leg \#3 has the highest average peak friction ( $1.24 \mathrm{gm}$ ) while it has second lowest cross angle ratio (see Table 7). Fig. 12 shows the crossing ratio vs. peak friction and it clearly demonstrates what has been described above. This is clearly explained by the familiar equation (13) for friction between two smooth bodies: $f=\operatorname{Ar} \cdot \tau$ where $f$ is the coefficient of friction, $A r$ is the real area of contact and $\tau$ is the shear strength of the asperity contacts. Thus, the smoother the interface between the head and the disk the higher the real area of contact; therefore, the higher the friction forces. One way to prevent the high initial friction force is to increase the initial surface roughness of the disk. From Table 6, leg \#6 with initial Ra of 56.3 A (highest among 9 legs) exhibits the lowest average friction force, while leg \#7 with second lowest Ra 
(42.5 A) in the group displays the highest friction force. This result again strongly supports the friction equation. TABIE 5. BUDLARY OF DRNG TB8T FOR DIFFERENY
TELTURING PROCE88E8

$\begin{array}{ccccc}\text { IEG } & & \text { USE. } & \text { UKF. } & \text { Umax. } \\ \text { PROCESS I } & \text { AVG } & 0.340 & 0.310 & 0.390 \\ & \text { STD } & 0.026 & 0.009 & 0.014 \\ \text { PROCESS II } & \text { AVG } & 0.363 & 0.315 & 0.423 \\ & \text { STD } & 0.025 & 0.005 & 0.023 \\ \text { PROCESS III AVG } & 0.368 & 0.296 & 0.434 \\ & \text { STD } & 0.020 & 0.015 & 0.021\end{array}$
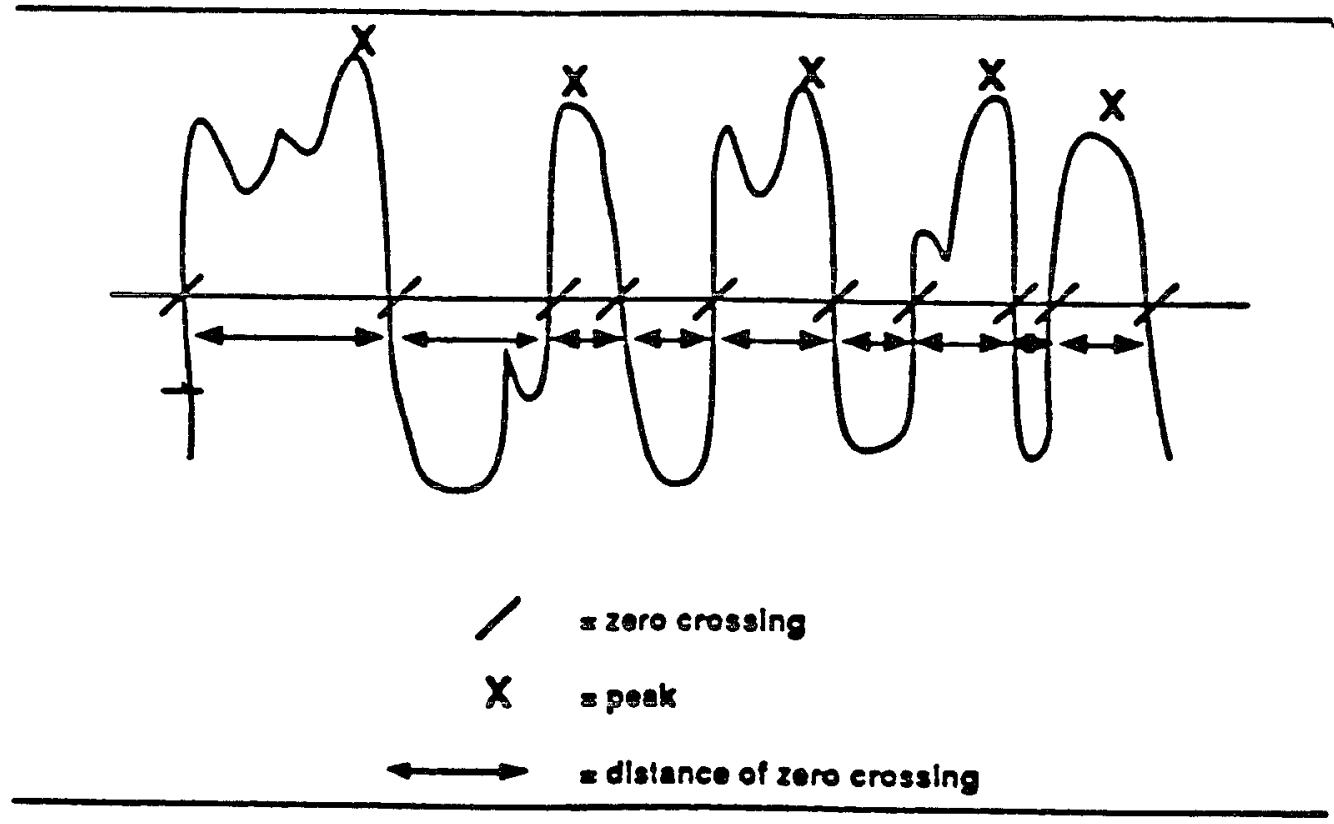

Definition of 20 peaks aod crossings.

Figure 10. Graphical Definition of Zero Crossing and 2-D Peaks. (Wyko) 
Top view of disk surface
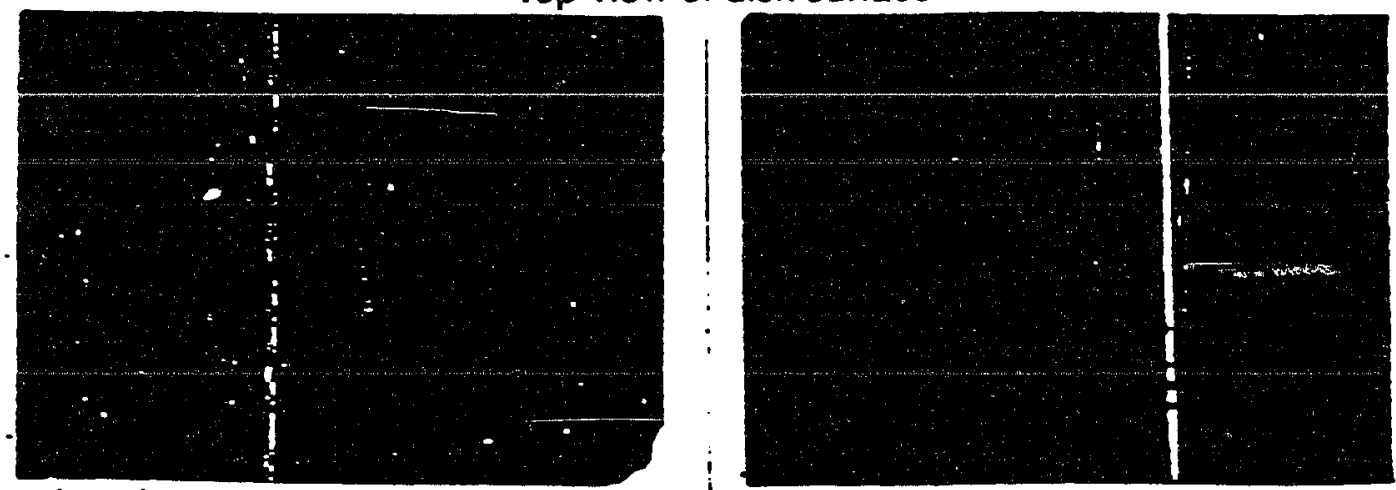

$\log 1$ $200 x$

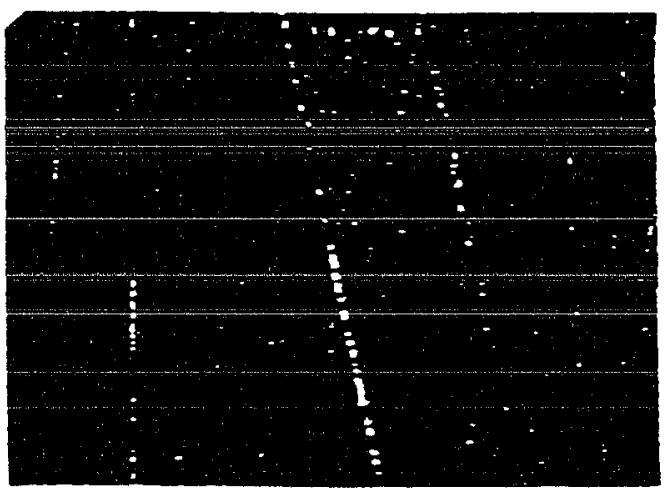

$200 x$
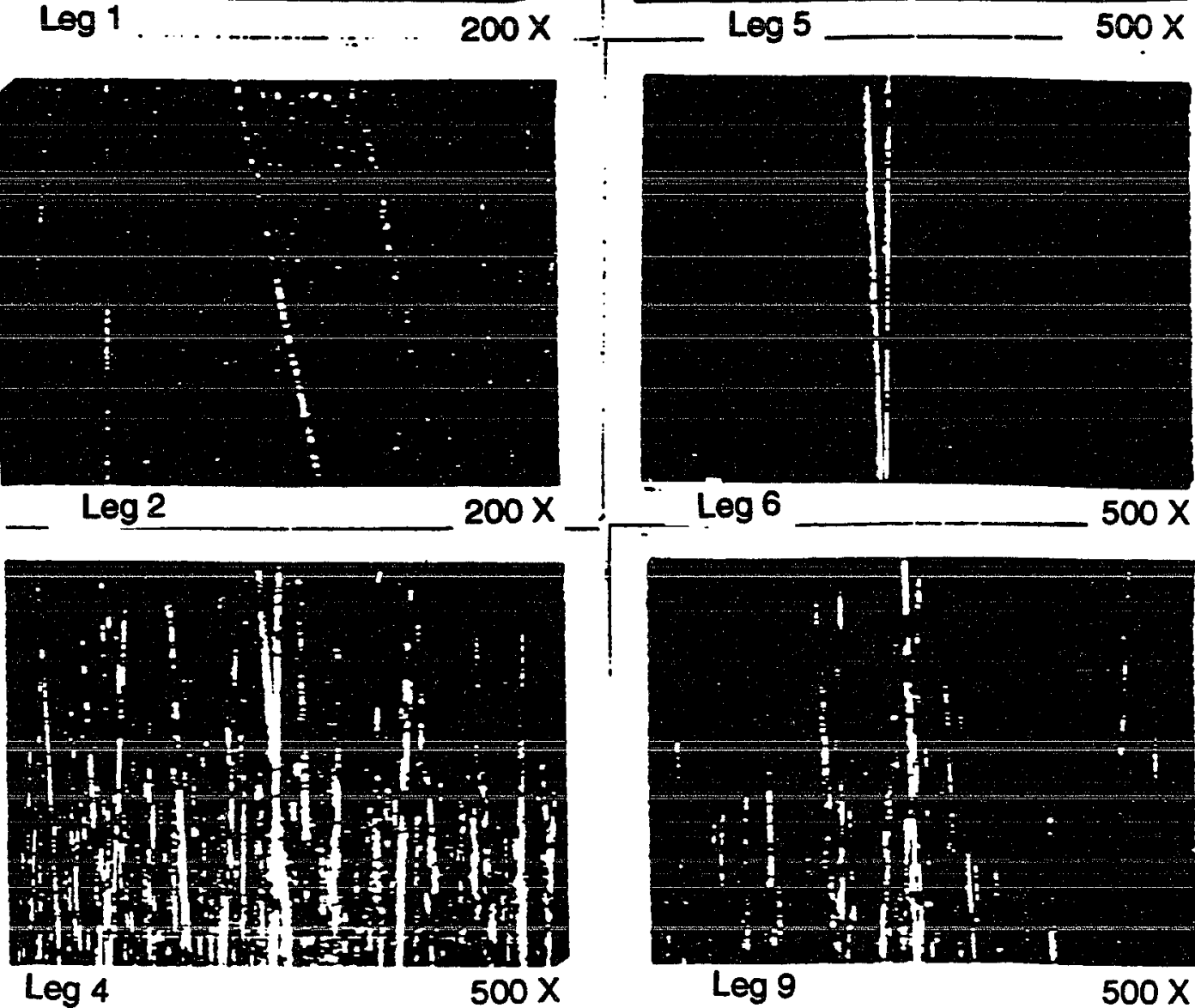

$\operatorname{Leg} 6$ $500 x$

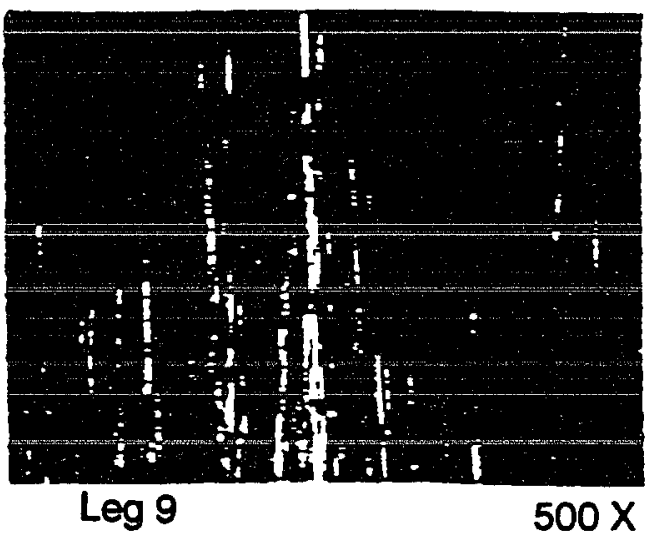

Figure 11. High Magnification Pictures for Various Legs. 


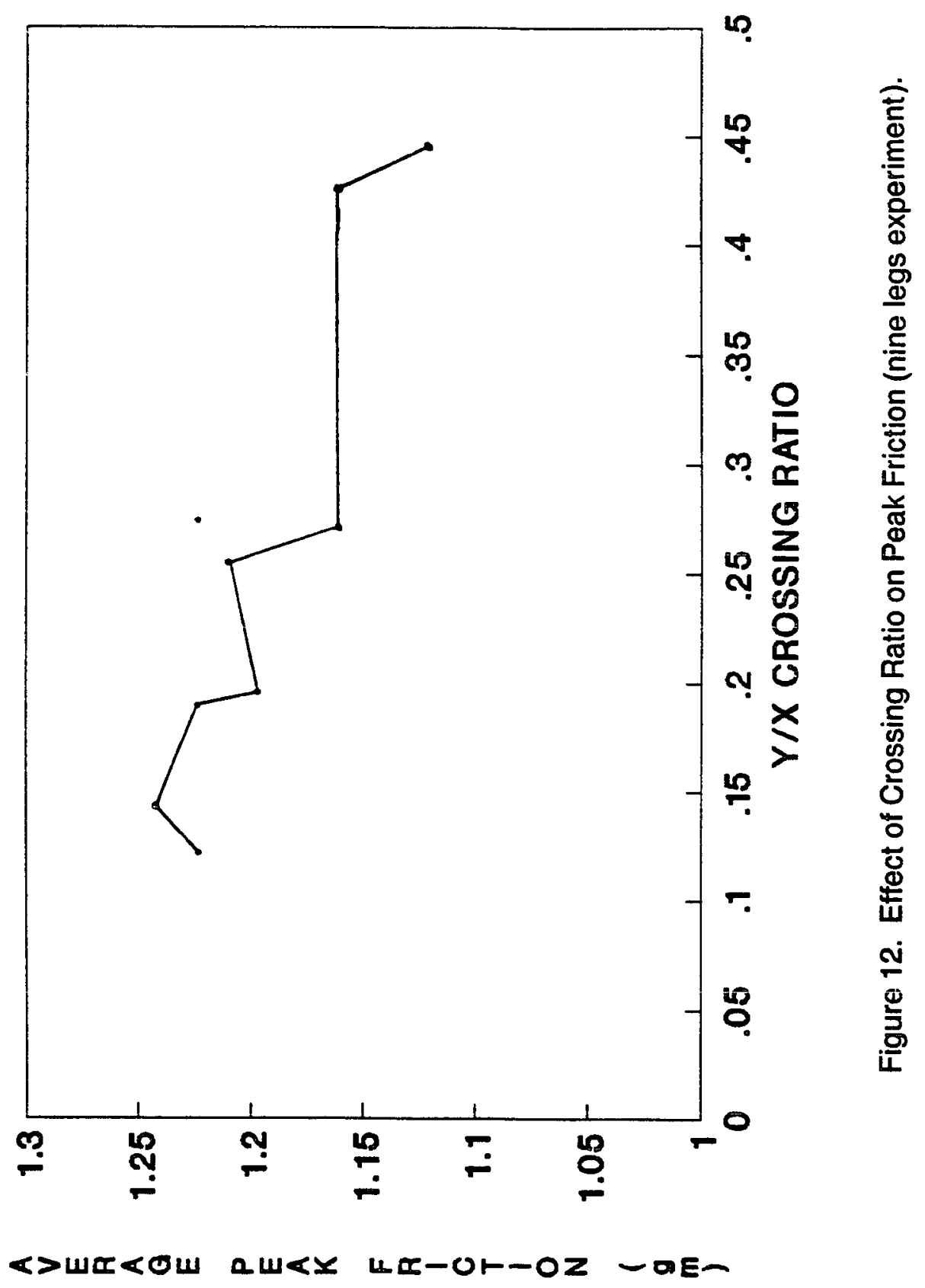




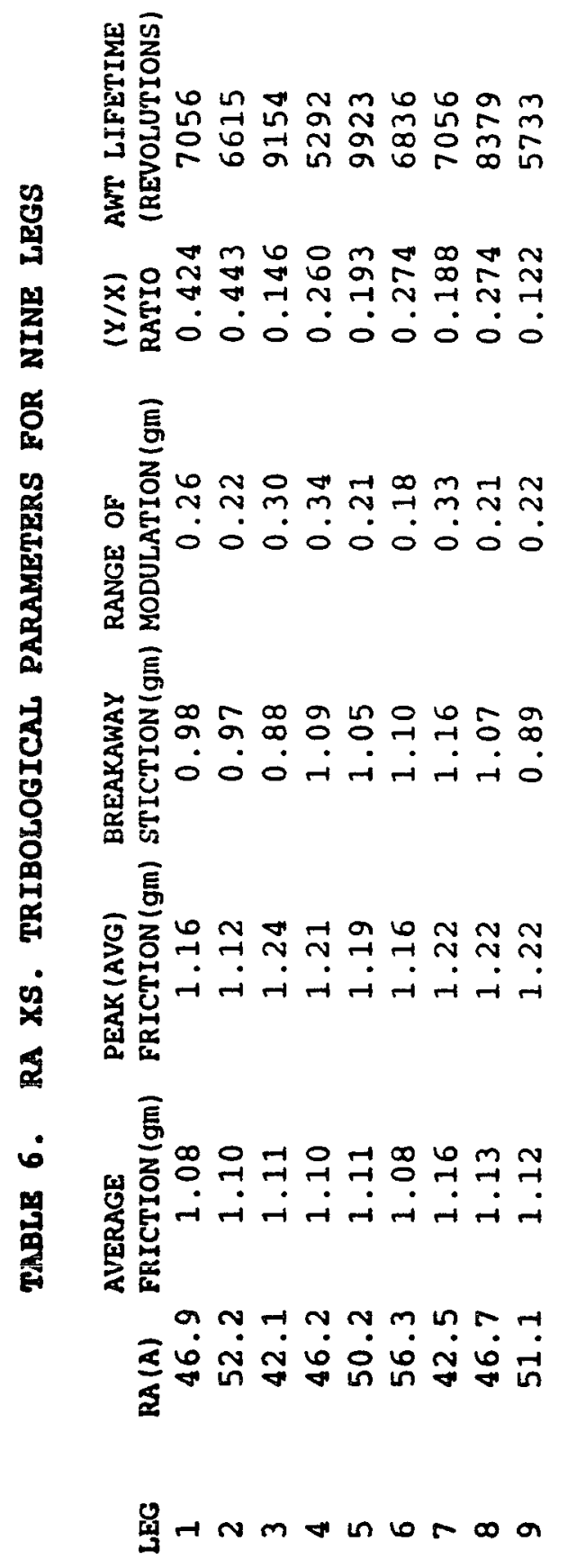




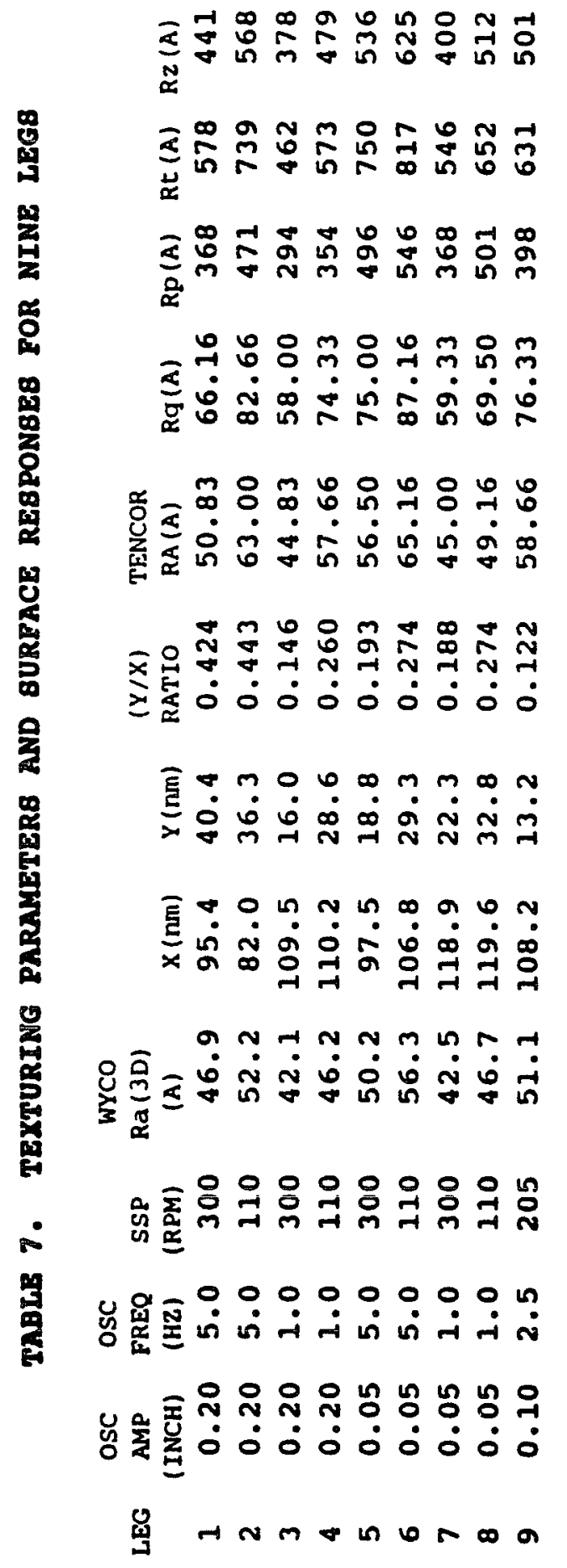


Another parameter called the range of modulation is also obtained from this EDC 1RPM test. The range of modulation is defined as the calculated delta between the maximum friction and the minimum friction from the friction test. This parameter is usually an indication of how much fluctuation a specific texturing process possessed. The data of the range of modulation are summarized in Table 6. Fig. 13 shows the crossing ratio vs. the range of modulation for the nine legs experiment. No clear trend is indicated from this figure.

The results of the accelerated wear test for the nine legs matrix is summarized in Table 6 and in Figure 14. Due to lack of a dry buffing step after texturing, all nine legs exhibit very early failure. Although leg \#5 showed a little higher in average cycles before failure, it does not prove any significance over the other legs. One can not draw any conclusion on whether any specific leg exhibits better AWT characteristics. The nine legs experiment has been repeated with the dry buffing step added and the AWT results are shown in Table 8 and Figure 15. Each bar in Figures 14 and 15 represents a disk surface. Thus, double bars mean two surfaces have been tested while quadruple bars mean four surfaces were tested.

The repeated nine legs are more easily distinguished than in the case of previous experiment. Leg \#3 has the longest cycles before failure from AWT test followed by leg\#5 and leg\#8. On the other hand, leg \#1 and leg\#2 has the lowest cycles before failure. The texturing parameters and AWT cycles of failure for the repeated nine legs experiment are given in Table 8 , 


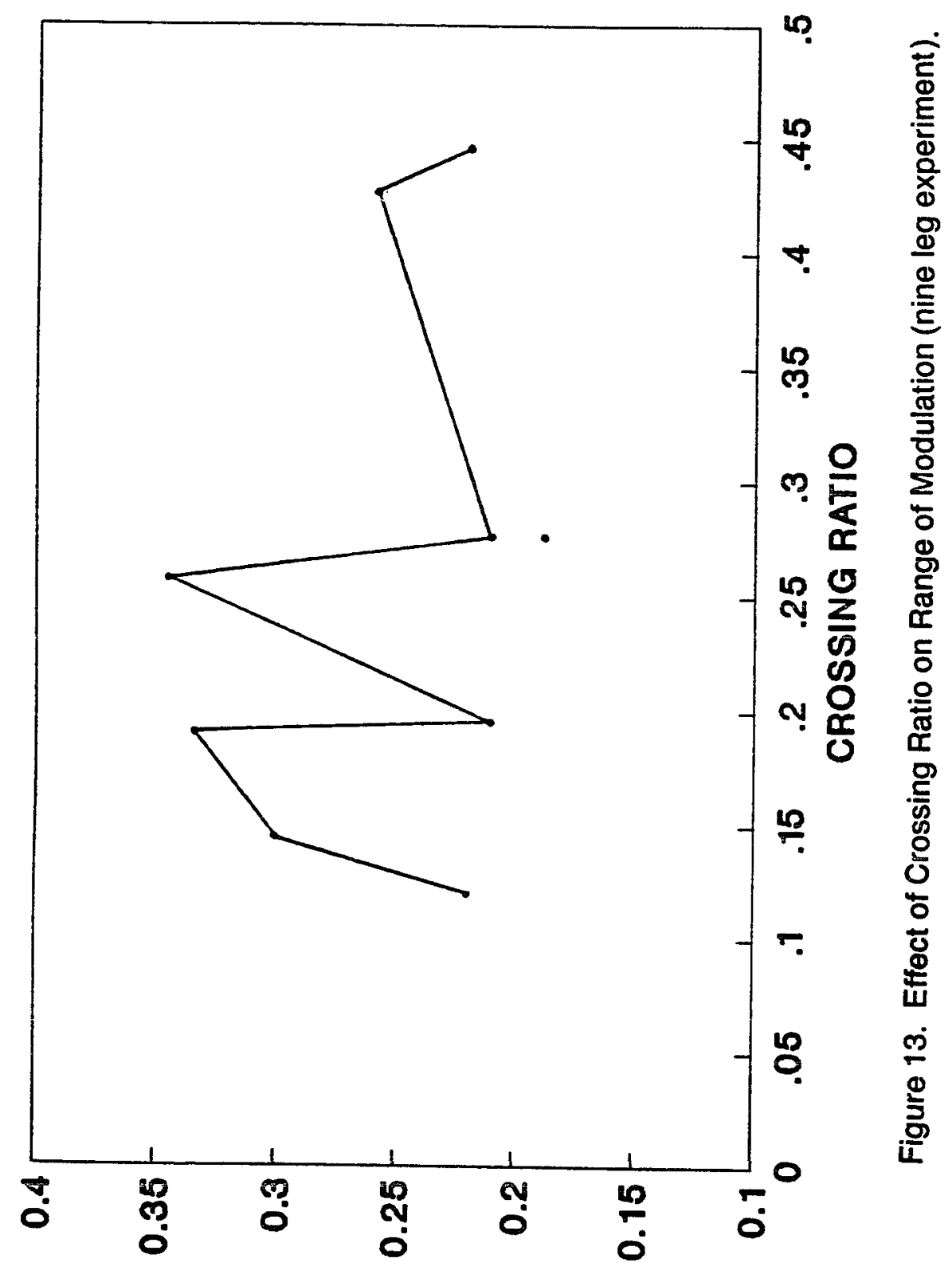

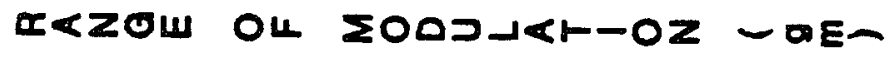




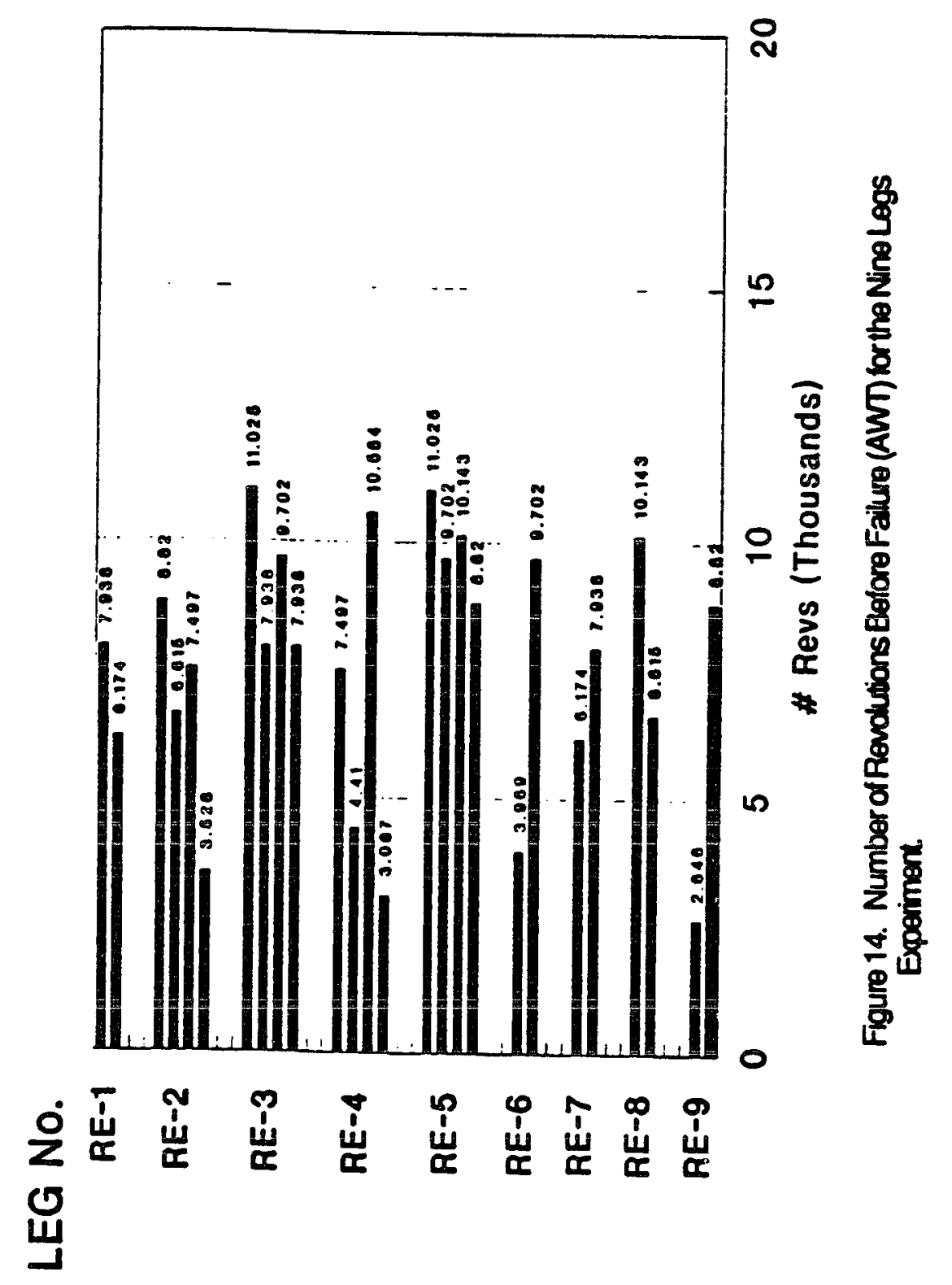




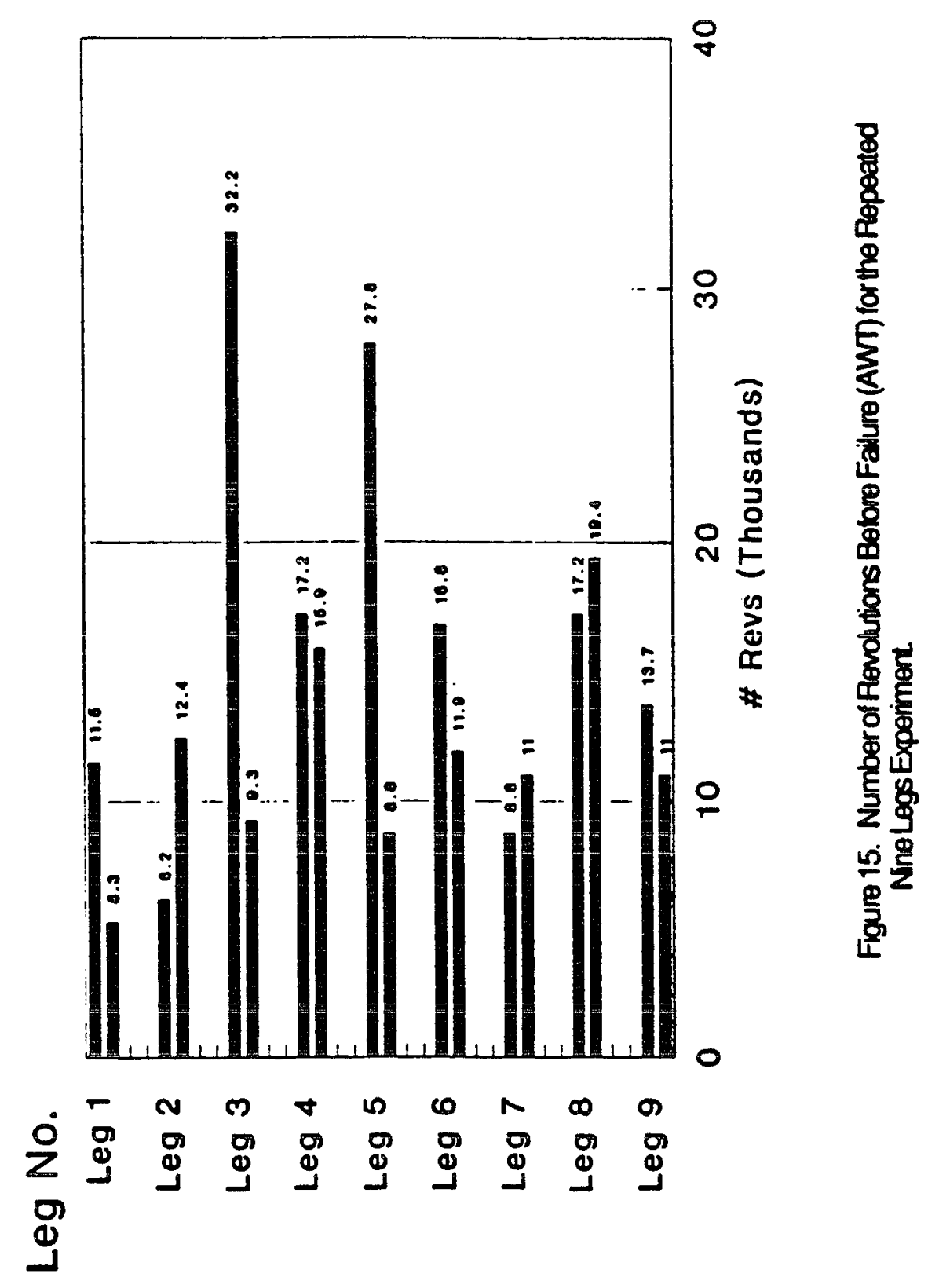




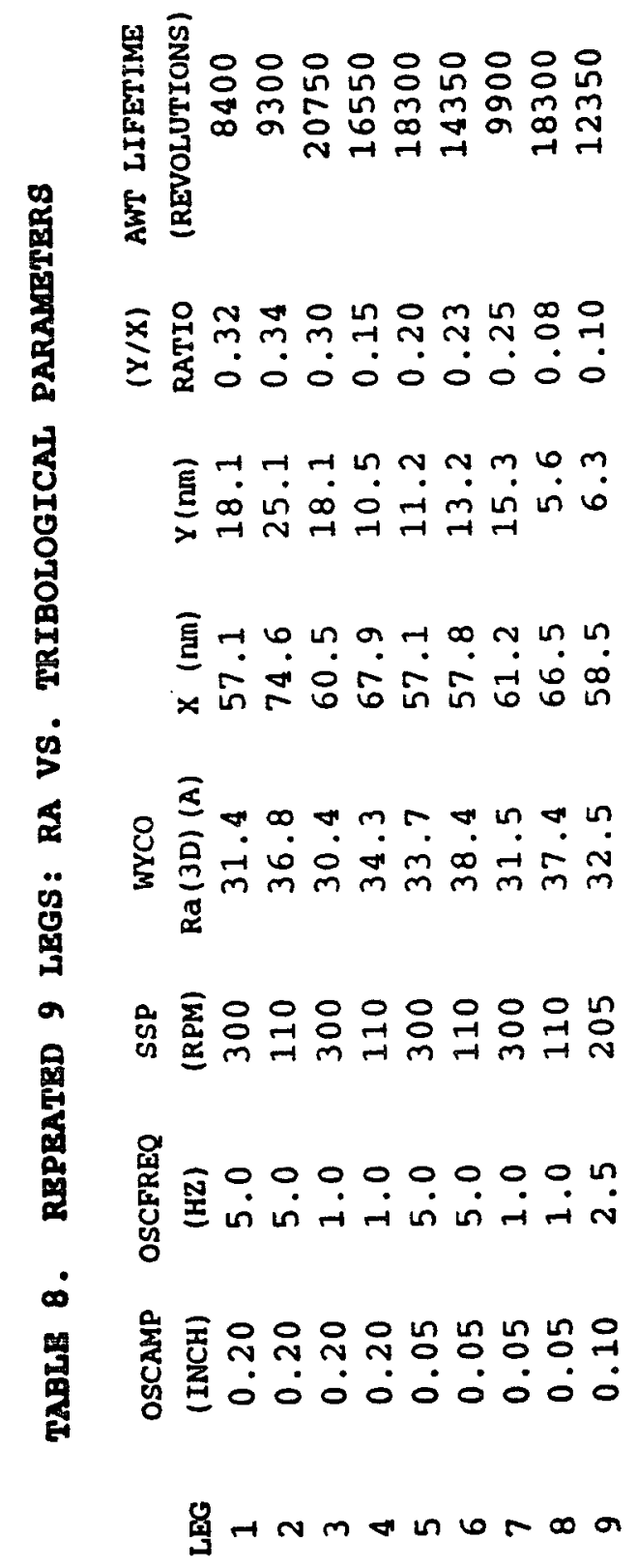


while Table 9 summarizes the data of crossing ratio vs. AWT failure cycles for both nine legs experiments.

TABLE 9. CROB8ING RATIO VB. AwT FAILURE

$\begin{array}{rcc}\text { AWT CYCLES REPEATED } 9 \text { LEGS } & 9 \text { LEGS EXPT. } \\ 0 & \text { RATIO }(Y / X) & \text { RATIO }(Y / X) \\ 8400 & 0.32 & 0.424 \\ 9300 & 0.34 & 0.443 \\ 9900 & 0.25 & 0.188 \\ 12350 & 0.10 & 0.122 \\ 14350 & 0.23 & 0.274 \\ 16550 & 0.15 & 0.260 \\ 18300 & 0.20 & 0.193 \\ 18300 & 0.08 & 0.274 \\ 20750 & 0.30 & 0.146\end{array}$


Figure 16 shows the crossing ratio vs. cycles before failure for the nine legs experiment while Fig. 17 shows the same plot for the repeated nine legs. These two figures indicate that the $y$ over $x$ crossing ratio is indeed inversely related to cycles before failure. Since the head loading force is constant regardless of how many surface peaks are present, the higher the crossing ratio, the more the peaks, and the lower the AWT cycles before failure.

A statistical software package was used to characterize the surface topography relationship. This software package is called Statgraphics. Statgraphics* enables one to determine the main significant factor of a designed experiment at a $95 \%$ confidence level. The $95 \%$ confidence level is defined as the probability that certain phenomena will exist 95 out of 100 times under a predetermined circumstance. For the three dimensional Ra roughness from Wyco for example, both oscillation frequency (OSCFREQ) and disk rotational speed (SSP) are considered significant. Table 10 summarizes these statistical values. The confidence level for OSCFREQ is $98.44 \%$ while for SSP it is $97.77 \%$. Both Figure 18 and Figure 19 show that while holding the SSP constant, 3D Ra will increase as the OSCFREQ increases. This can be explained that as the OSCFREQ increases, so does the relative movement between the disk and the abrasive tape. The additional rubbing thus increases the average roughness values. Another

* Dynamic Microsystems, 13003 Buccaneer Rd., Silverspring, M.D. 20904. 


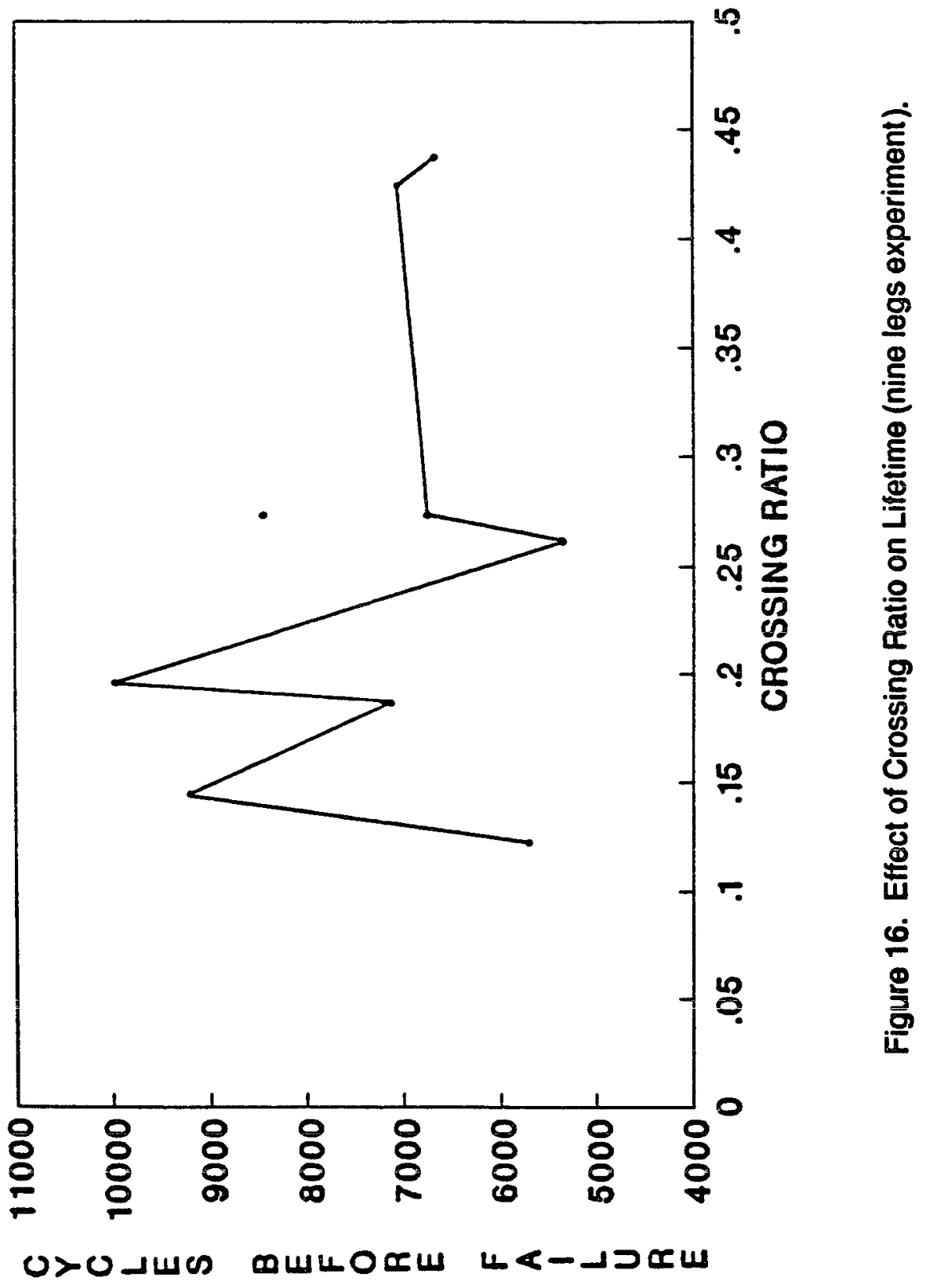




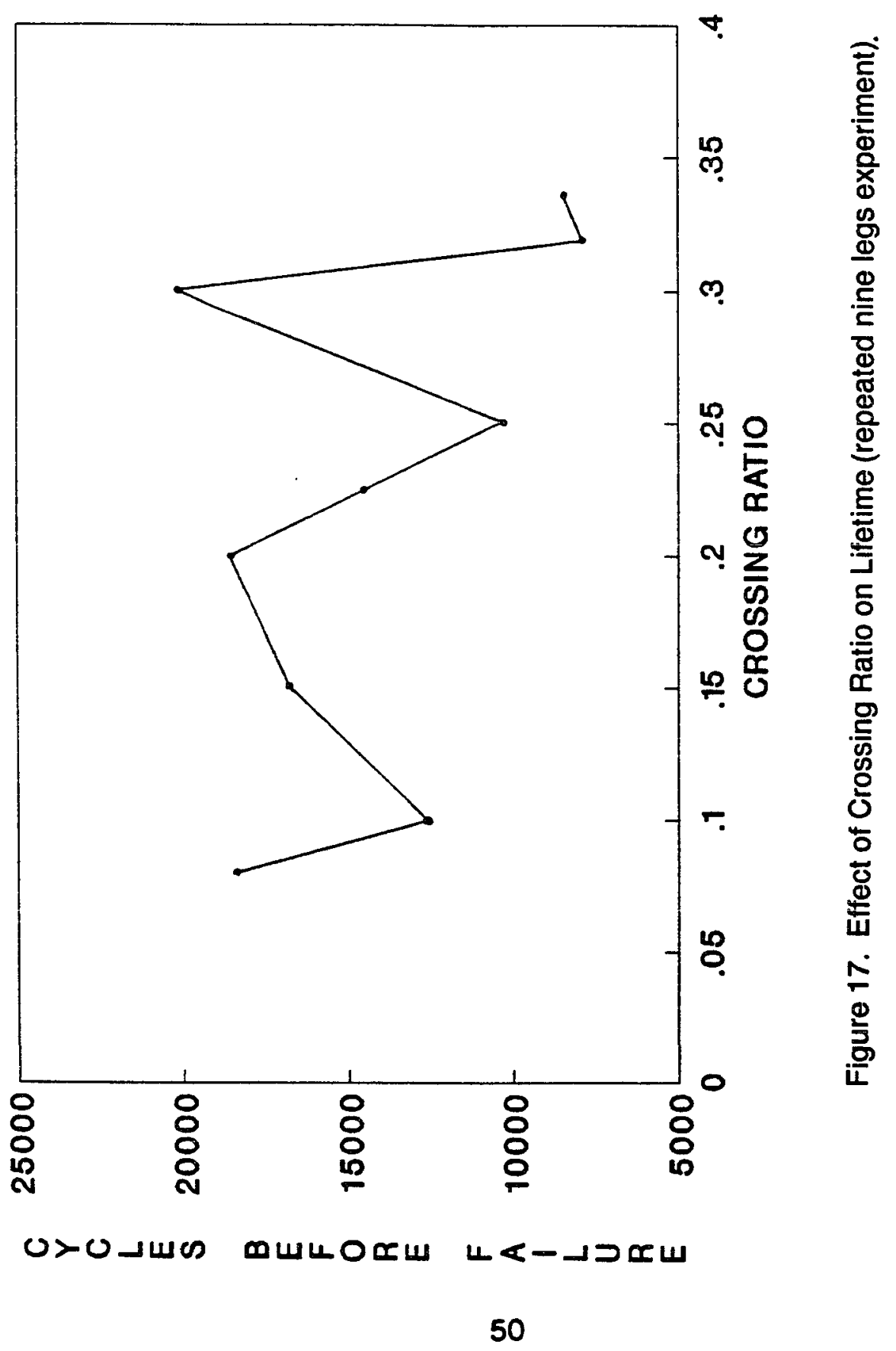




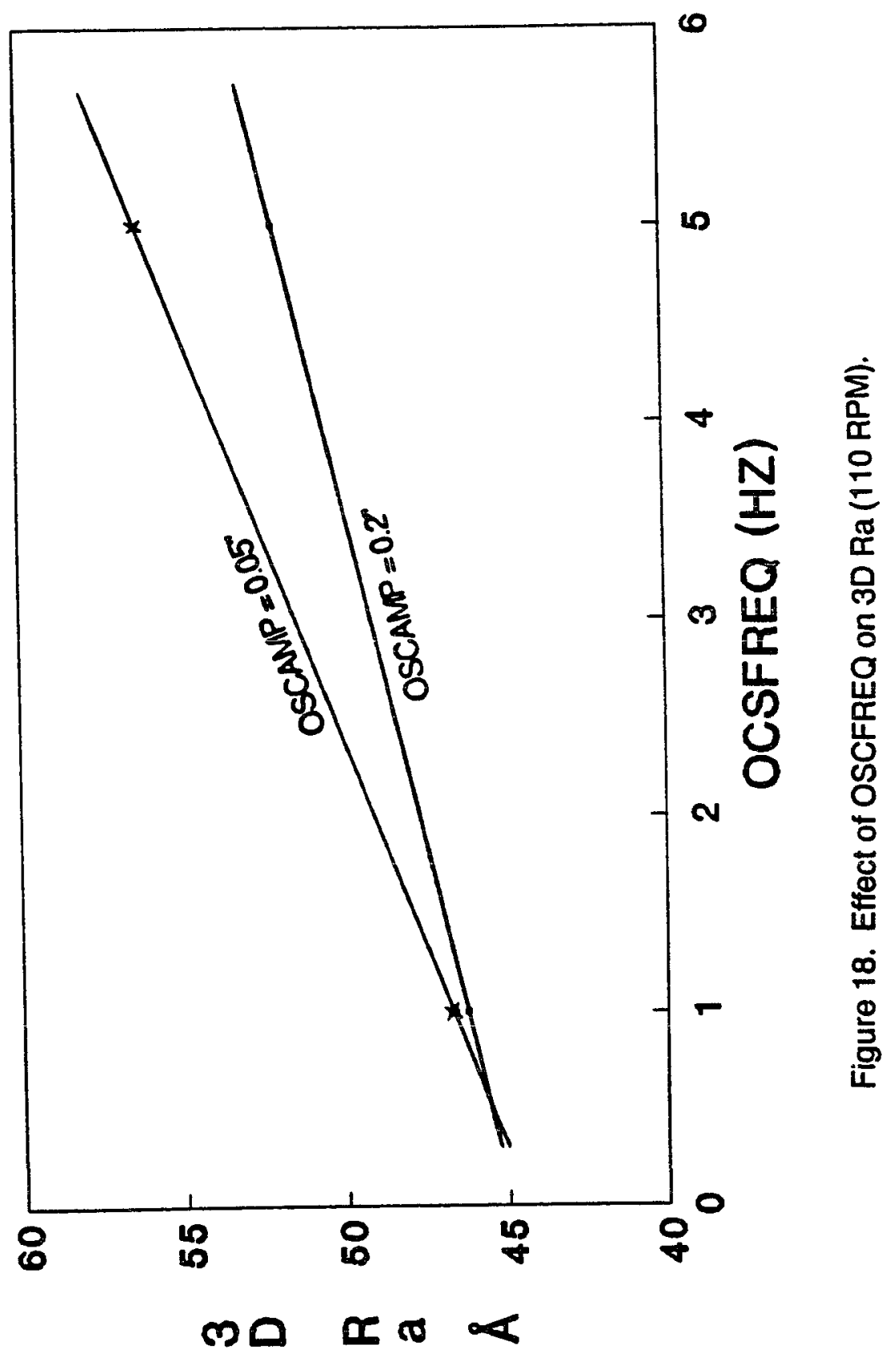




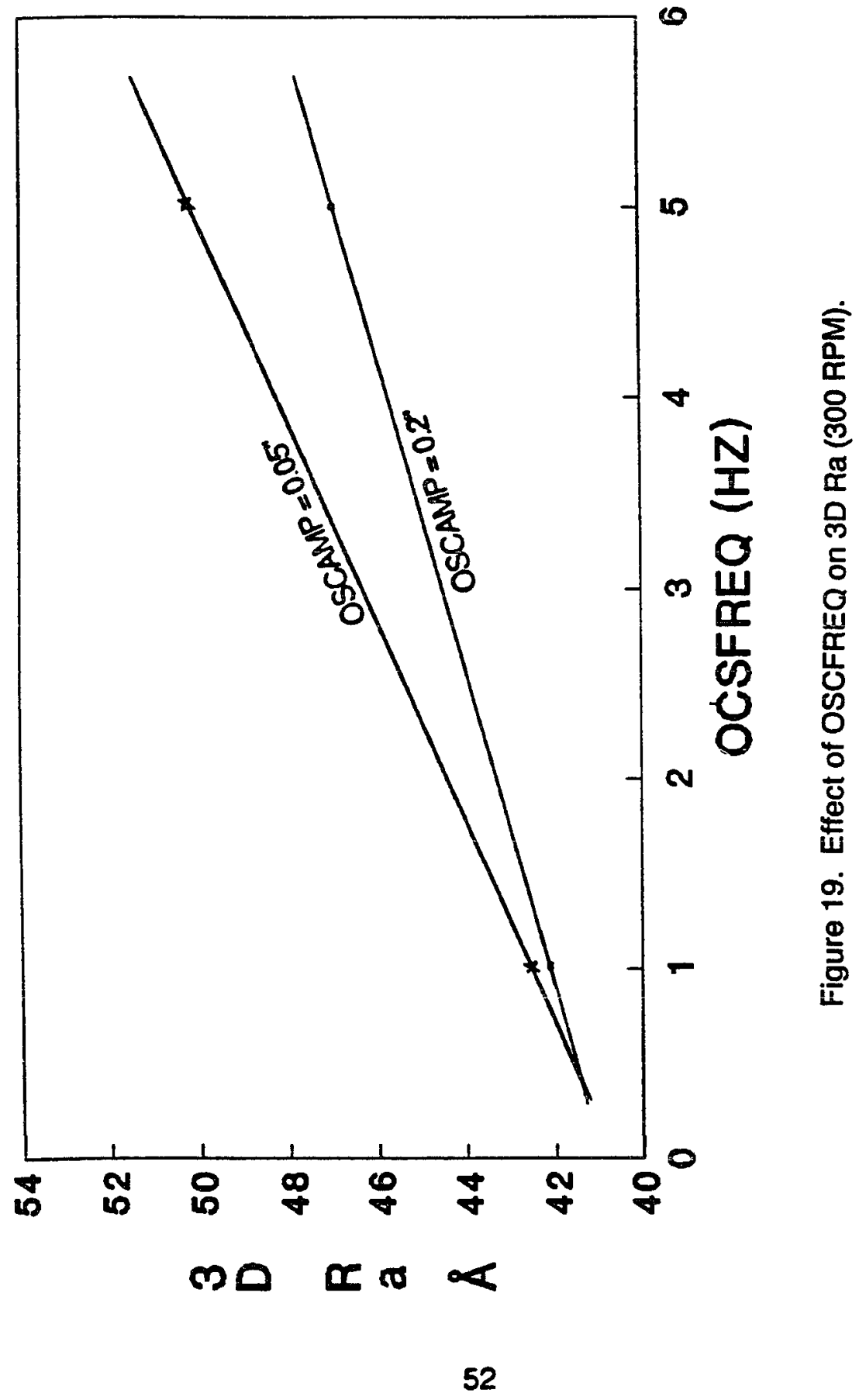


TABLE 10. ANALYSIS OF VARIAYCE FOR VARIOUS gURFACE IN THE NINE LEGS ITPERINIENT

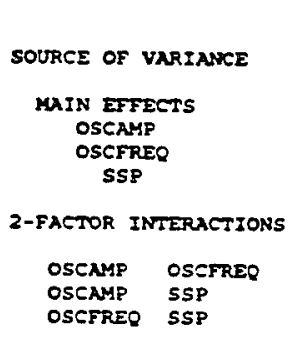

SOURCE OF VATIANCE

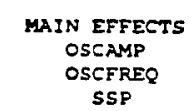

2-FACTOR INTERACTIONS

OSCAMP OSCFREQ

$\begin{array}{ll}\text { OSEAMP } & \text { SSP } \\ \text { OSTFREQ } & \text { SSP }\end{array}$

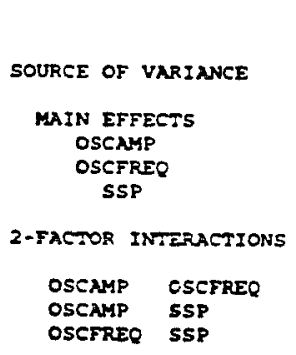

RaT10 $(Y / X)$

CONFIDENCE UEVIL

.8289
.8393
.8802
.8170
.7643
.8776
.2267
.5412

3D Ra (wTKO)

CONFIDDNCE LEVEL

$$
\begin{aligned}
& .9751 \\
& .9472 \\
& .9846 \\
& .9777 \\
& \\
& .8796 \\
& \\
& .9326 \\
& .5858 \\
& .8605
\end{aligned}
$$

Rq (TRNCOR)

CONFIDENCE LEVEL

.9695

\begin{tabular}{|c|c|}
\hline SOURCE OF VARIANCE & $\begin{array}{l}\text { RP (TENCOR) } \\
\text { CONF IDENCE LEVEL }\end{array}$ \\
\hline $\begin{array}{l}\text { MAIN EFFECTS } \\
\text { OSCAMP } \\
\text { OSCFREQ } \\
\text { SSP }\end{array}$ & $\begin{array}{l}.7650 \\
.8186 \\
.7908 \\
.7808\end{array}$ \\
\hline 2-FACTOR INTERACTIONS & .0202 \\
\hline $\begin{array}{ll}\text { OSCAMP } & \text { OSCFREQ } \\
\text { OSCAMP } & \text { SSP } \\
\text { OSCFREQ } & \text { SSP }\end{array}$ & $\begin{array}{l}.0891 \\
.0989 \\
.193\end{array}$ \\
\hline
\end{tabular}

.8851
.9771

.9793

.8652

.9324

.9324
.8922

.8922
.5614
RE (TENCOR)

CONFIDENCE LEVIL

SOURCE OF VAF:ANCE
MAIN EFFECTS
OSCAMP
OSCFREQ
SSP
2-FACTOR INTERACTIONS
OSCAMP OSEFREQ
OSCAMP SSP
OSCFREQ SSP

SOURCE OF VARIANCE

MAIN EFFECTS
OSCAMP
OSCFREO
SSP

2-FACTOR INTERACTIONS

OSCAMP CSCFREQ

OSCAMP SSP

OSCFREP SSP
.8752 .8667
.9187 .9187
.876

.328

.4844

.5415

.0772

$R 2$ (TENCOR)

CONFIDENCE LEVEL

.9016

.854

.9236

.9286

.4697

.7065

.3159

$.03 \mathrm{BA}$ 
main factor, oscillation amplitude (OSCAMP), is somewhat significant; even though its level of confidence $(94.72 \%)$ is not quite $95 \%$. Figure 20 and Figure 21 show that while holding the OSCFREQ constant, 3D Ra will decrease as the SSP increases. The reason is probably that when the SSP increases the interfacial contact between the tape and the disk will decrease. This phenomenon prevents the relative movement between the disk and the abrasive tape. The reduced rubbing thus decreases the average roughness values. Besides the single main factor, the interaction of any two main factors can also be significant. The two interaction factors of OSCAMP and OSCFREQ with confidence level of $93.26 \%$ is somewhat significant also. The other two interactions (OSCAMP, SSP) and (OSCFREQ, SSP) showed no significant contribution to the Ra roughness. 


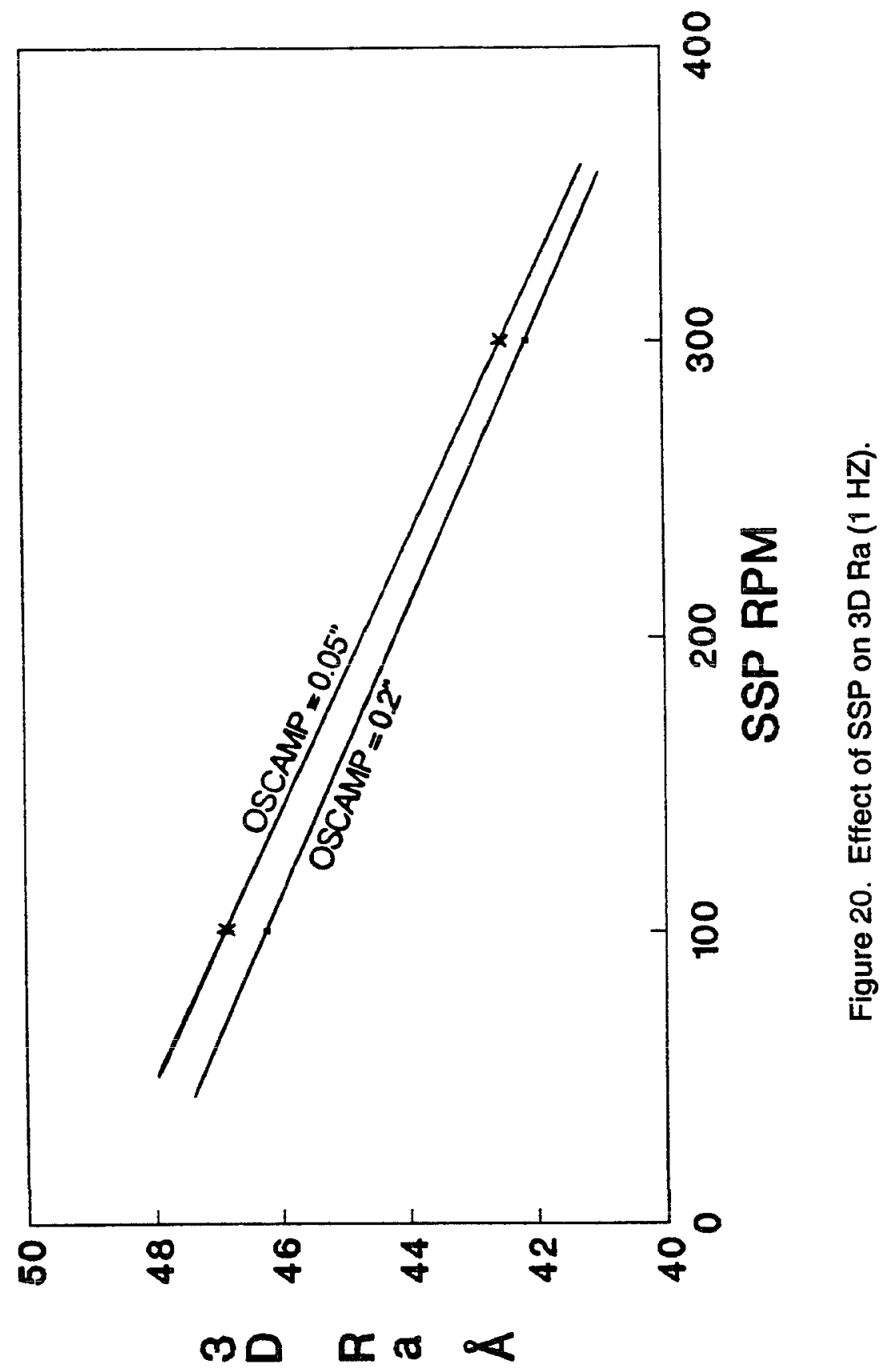




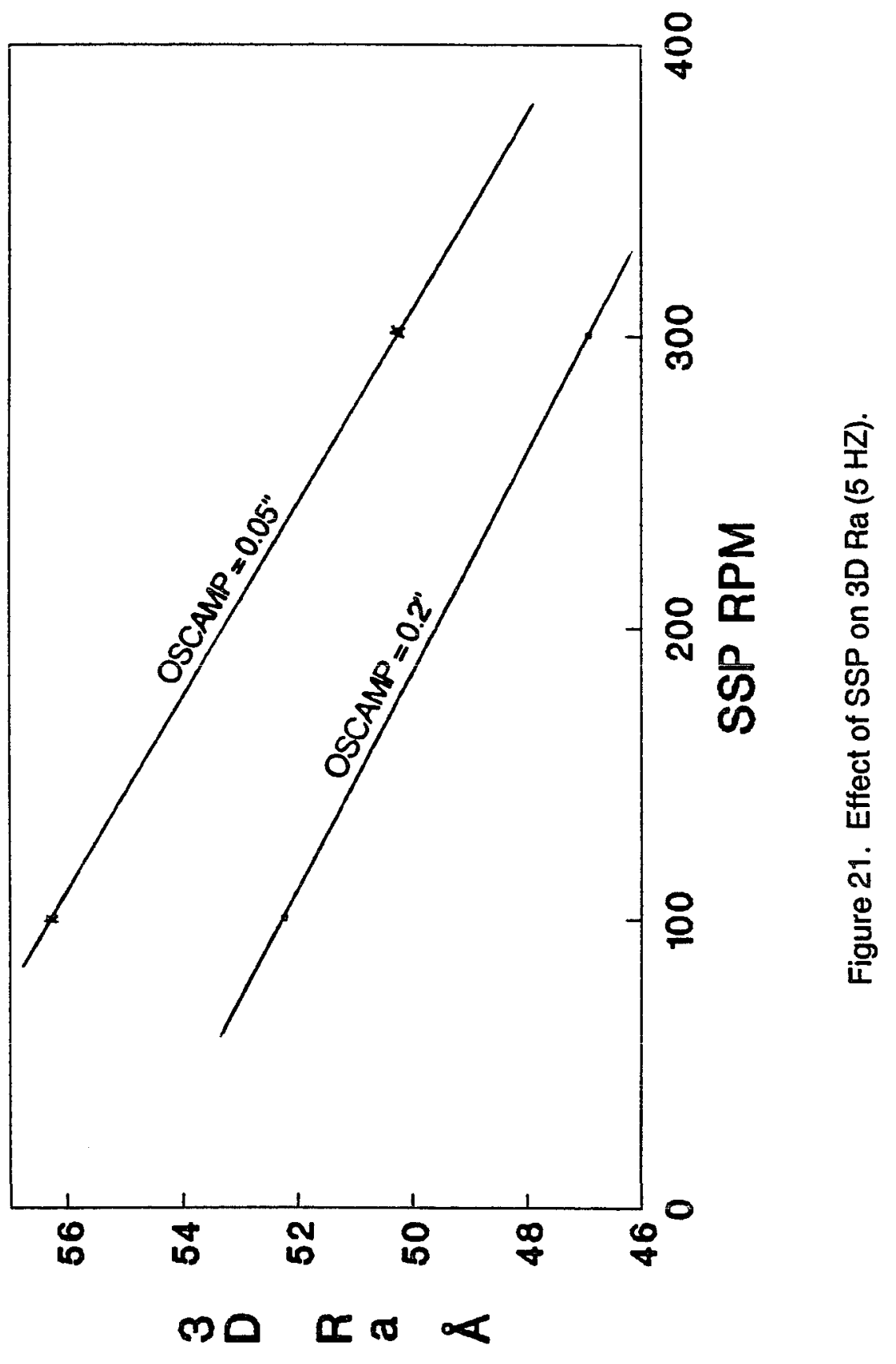




\section{CONCLUSION}

The experimental data show that as the crossing ratio increases the friction decreases. This is desirable for a good texturing process. On the other hand, when the crossing ratio increases the AWT cycles before failure also decrease. From this result, it may be inferred that the head-disk interface will not last for the desired number of revolutions in a rigid disk drive under normal operating conditions. This is an obvious trade off situation that needs to be carefully dealt with. Additional research is required in optimizing the crossing ratio in order to satisfy both friction and wear operating windows. Dry buffing is an important step in the manufacturing of rigid thin film disks. However, the extent of dry buffing must be closely controlled in order to achieve maximum efficiency. To obtain a good glide yield a more moderate abrasive tape is recommended with the aid of dry buff. The 3D Ra can be easily modified by controlling the SSP and OSCFREQ parameters in order to provide desired values for head/disk interface response. 


\section{REFERENCES}

1) Trauner, D., Li, Y. and Talke, F. E., "Frictional Behavior of Magnetic Recording Disks," IEEE Trans. Mag., Jan. (1990).

2) Doan, T. Q. and MacKintosh, N. D., "The Frictional Behavior of Rigid Disk Carbon Overcoats," Trib. and Mech. of Magnetic Storage Syst., SP-25, pp 611 (1988).

3) Marchon, B., Vierk, S., Heiman, N., Fisher, R. and Khan, M., "Significance of Surface Roughness Measurement Application to the Tribology of the Head/Disc Interface," Trib. and Mech. of Magnetic Storage Syst., SP-26, pp7180 (1989).

4) Walmsley, R. G., Brandt, J. and Natarajan, B. R., "Detection of Failure in Disk Wear Testing," Trib. and Mech. of Magnetic Storage Syst., SP-26, pp 128131 (1989).

5) Marchon, B., Heiman, N. and Khan, M., "Evidence for Tribochemical Wear on Amorphous Carbon Thin Films," IEEE Trans, Mag., Jan. (1990).

6) Suk, M. and Bogyu, D. B., "Dynamics and Wear of the Head-Disk Interface During Constant Speed Sliding," Technical Report 18, Center of Magnetic Recording Research, UC at San Diego (1988). 
7) Bowden, F. P, and Tabor, D., Friction and Lubrication of Solids, Part II, Clarendon Press, Oxford (1964).

8) Suzika, S. and Kennedy, F. E., "Friction and Temperature at Head-Disk Interface in Contact Start/Stop Tests," STLE, SP-25, pp 30-36 (1988).

9) Yeack-Scranton, C. E. "Novel Piezoelectric Transducer to Monitor HeadDisk Interactions," IEEE Trans. Mag., MAG-22, No. 3, pp 1011-1016 (1986).

10) Tseng, R. C. and Talke, F. E., "Transition from Boundary Lubrication to Hydrodynamic Lubrication of Slider Bearings," IBM J. of Research and Development, Vol. 16, No 3, pp 534-540 (1974).

11) Gatzen, H. H., Smallen, M. J., and Tedrow, P. T., "Head-Media Wear in 5.25 Inch Disc Drives," STLE, SP-22, pp 116-122 (1987).

12) Kita, T., Kogure, K., Mitsuya, Y., and NaKaishi, T., "New Method of Detecting Contact Between Floating-Head and Disk, "IEEE Trans. Mag., MAG16. No. 5, pp 873-875 (1980).

13) Benson, R. C., Sundaram, R., and Talke, F. E., "A study of Acoustic Emission from the Slider-Disk Interface in a 5.25 Inch Hard Disk Drive," STLE, SP-25, pp 87-93 (1988). 
14) Rabinowicz, E., "The Tribology of Magnetic Recording Systems-An Overview," Trib. and Mech. of Magnetic Storage Syst., ASLE SP-21, Pp 1 (1986).

15) Timisit, R. S. and Stratford, G., "Effect of Humidity on Friction at MagneticHead/Hard-Disk Interfaces," Trib. and Mech. of Magnetic Storage Syst., STLE, SP-25, pp 17-23 (1988).

16) Teng, E. and Ballard, N., "Anisotropy Induced Signal Waveform Modulation of DC Magnetron Sputtered Thin Film Disks, "IEEE Trans. Mag., MAG-22, pp 579 (1986).

17) Seagle, D., Fernelius, N., and Khan, M., "Influence of Substrate Texture on Recording Properties for CoNiCr Rigid Disk Media," Presented at Magnetism and Magnetic Matl's Conf. (1986).

18) Liu, C. C. and Mee, P. B., "Stiction at the Winchester Head-Disk Interface," IEEE Trans. on Mag., MAG-19, pp 1659-1661 (1983).

19) Judge, J. S. and Speliotis, D. E., "The Effect of Texturizing on the Magnetic and Recording Properties of Plated Rigid Disks," IEEE Trans. on Mag., MAG-23, pp $3402-3404$ (1987).

20) Bate, G., "Recent Developments in Magnetic Recording Materials," J. Appl. Phys., 52 (3), pp 2447-2451, March (1981). 
21) Raman, V. Tang, W. T. Jen, D. and Reith, T. R., "The Dependence of Stiction and Friction on Roughness in Thin-Film Magnetic Recording Disks," J. Appl. Phys., 70 (3), pp1826-1836, August (1991).

22) Tsai, H. C., Mehmandoust Y., Samani H., and Eltoukhy A., "Tribological Phenomena at the Head-Disk Interface of Thin-Film Rigid Disks," J. Vac. Sci. Technol. A7 (3), May/Jun (1989).

23) Eltoukhy, A. H., "A Review of Thin Film Media for Magnetic Recording," J. Vac. Sci. Technol. A 4 (3) May/June (1986).

24) White, R. M., "Disk-Storage Technology," Sci. Amer., Vol. 243, pp 138-148, Aug. (1980).

25) Tsuya, N, Tokushima, T., Shiraki, M., Wakui, Y., Saito, Y., Nakamura, H., and Harada, Y., "Micro-Tribology of Alumite Disc using Anodic Oxidation," IEEE Trans. Mag., MAG-24, No. 6, pp 2661-2663 (1988).

26) Day, C. K. HarKins, C. G., Howe, S. P., and Poorman, P., "Thin-Film Disc Reliability- the Conservative Approach," HP Journal, pp 25-28, Nov. (1985).

27) Chang, W. R., Etsion, I., Bogy, D. B., "Static Friction Coefficient Model for Metallic Rough Surfaces," Journal of Tribology, Vol. 110, pp 57-62, Jan. (1988). 
28) Bhushan, B., Wyant, J. C., Koliopoulos, C. L., "Measurement of Surface Topography of Magnetic tapes of Mirau Interferometry," Applied Optics, Vol. 24, No. 10, 15 May (1985).

29) Robinson, G. M., Perry, D. M., and Peterson, R. W., “Optical Interferometry of Surfaces," Scientific American, pp 66-71, July (1991).

30) Streator, J. Bogy D. B., Etsion, I., "The Effect of Lubrication on the Static and Low-Speed Dynamic Friction in Thin Film Magnetic Disks," Tribology and Mechanics of Magnetic Storage System, 5, STLE, SP-25, pp 24 (1988).

31) Chu, M., Bhushan, B., and DeJonghe, L. C., "Wear Behavior of Ceramic Sliders in Sliding Contact with Rigid Magnetic Thin-Film Disks," pp 9-16 (1988).

32) Bhushan, B., "Magnetic Media Tribology: State of the Art and Future Challenges," Wear, 136, pp 169-197, (1990).

33) Lemke, J. U., "Physical Limits of Magnetic Recording," Tutorial Magnetic, Center for Magnetic Recording Research, UCSD, pp 1-7.

34) Bertram, H. N., "Geometric Effects in the Magnetic Recording Process," IEEE Trans. Mag., MAG-20, No. 3, May (1984). 
35) Miller, P. C., "Texture Choices: What Kind of Surface Do You Really Want?", Tooling \& Production, pp 46-48, June (1987).

36) Pratt \& Whitney Co Inc., "A System for Surface Roughness Characterization," Tooling \& Production, pp 43-46, June (1987).

37) Drews, W., and Weniger, W., "Rediscovering the Abbott-Firestone Curve," Quality, pp 50-53, Sept. (1989).

38) Krishnan, C., "Media Implications of Ultra-Low Flying Height Head/Disk Interfaces," Diskcon'89, September 26, 1989.

39) Bhushan, B., Doerner, M. F., "Role of Mechanical Properties and Surface Texture in the Real Area of Contact of Magnetic Rigid Disks, J. Trib. Trans. ASME, 111, pp 452-458 (1989).

40) Gatzen, H. et al, "Head-Media Wear in 5.25" Rigid Disk Drives," Trib. Mech. of Magnetic Storage Systems, 4, STLE, SP-22, October 1987.

41) Miyamoto,T., Sato, I., and Ando, Y., "Friction and Wear Characteristics of Thin Film Disk Media in Boundary Lubrication," Trib. Mech. of Magnetic Storage System, 5, STLE, SP-25, October 1988.

42) Kaneko, R., "Micro-Tribological Approach to Head-Medium Interfaces of Magnetic Recording," Proc. Eruotrib. 89, pp 210-215 (1989). 
43) Yamaguchi, H., Tsukamoto, Y. and Yanagisawa, M., "Mechanical Properties and Durability of Sputtered Carbon Protective Overcoats for Rigid Magnetic Disks," STLE Special Publication SP-25, pp 82.86 (1988).

44) Stout, K. J., "Surface Roughness: Measurement, Interpretation and Significance of Data," Materials in Engineering, 2, pp 260-295 (1981).

45) Ghabrial, S. R., and Zaghlool, S. A., "The Effect of Suriace Roughness on Static Friction," Int. J. Mach. Tool Des. Res., Vol 14, pp 299-309, 1974.

46) B. Bhushan, "Assessment of Accelerated Head-wear Test Methods and Wear Mechanisms," in B. Bhushan and N. S. Eiss (eds), Trib. and Mech. of Magnetic Storage Syst., SP-19, ASLE, Park Ridge, IL, pp 101-111, 1985.

47) Bhushan, J. C. Wyant and J. Meiling, "A New Three-Dimensional Digital Optical Profiler, Wear, pp 301-312, 122 (1988).

48) Y. Kawakubo, K. Ishihara, H. Seo and Y. Hirano, "Head Crash Process of Magnetic Coated Disk during Contact Start/stop Operations, IEEE Trans. Magn., Mag-20, pp 933-935, (1984).

49) Aronov, V., D'Souza, A.F., Kalpakyian, S., and Shareef, I., "Interations Among Friction, Wear, and System Stiffness - Part 2: Vibration Induced by Dry Friction," ASME Journal of Tribology, Vol 106, 59, (1984b). 
50) Chang, W. R., Etsion, I., Bogy, D. B., "An Elastic-Plastic Model for Contact of Rough Surfaces," ASME Journal of Tribology, Vol 109, 257, (1987).

51) Anonymous, "Surface Texture (surface roughness, waviness, and lay)", ANSI/ASME. B46.1, ASME, New York, (1985b).

52) N. Bertram, In "Magnetic Recording Technology" (Lecture Notes, Center of Magnetic Recording Research, University of California, San Diego, La Jolla, California, 1984). 


\section{APPENDIX}

Texturing process - The process in which the surface of the rigid disk is intentionally roughened by an abrasive tape.

Groove - A long narrow channel on the nickel-phosphorus surface caused by mechanical depression of an abrasive particle.

Tribology - The study of phenomena and mechanisms of friction, lubrication, and wear of surfaces in relative motion.

Surface texture - The repetitive or random deviation from the nominal surface that forms the three-dimensional topography of the surface.

Profile - The profile is the contour of the surface in a plane perpendicular to the surface, unless some other angle is specified.

Center Line - This line is the best-fit straight line drawn through the surface profile so that the total area of the profile above the line is equal to the area below the line.

Peak - A peak is the point of maximum height on that portion of a profile that lies above the center line and between two intersections of the profile with the center line.

Valley - A valley is the point of maximum depth on that portion of a profile that lies below the center line and between two intersections of the profile with the center line.

Peak-to Valley Height - The peak-to-valley height is the maximum excursion above the center line plus the maximum excursion below the center line within 
the sampling length.

Ra - The average of the absolute values of the deviation of the surface profile from the center line.

3D Ra - The three dimensional Ra values measured from Wyko profilometer.

Rq - The root-mean-square deviation of the profile from the center line.

Rp - The mean-to-peak values of a profile are the distances between the highest asperities and the center line.

Rv - The mean-to-valley values of a profile are the distances between the lowest valleys and the center line.

Rz - The distance between the average of five highest asperities and the five lowest valleys.

Zero Crossing - The point crossing the center line in the two dimensional topography of the surface.

Friction Force - A force which resists the motion of two solid bodies that slide relative to one another.

Maximum Static Friction (MSF) - The maximum tangential force which is attained just before the head breaks free from the disk.

Kinetic Friction Force (KF) - The tangential force which maintains the sliding at the head-disk interface.

Stiction - An anomalously large static friction force, often due to wetting of the head/disk interface by lube which accumulates at the interface by capillary 
attraction. Stiction may be aggravated by long contact time and by high humidity environment.

Wear - Removal of material as a result of friction.

Coefficient of Static Friction $\left({ }_{\mathrm{Sf}} \mathrm{f}\right)$ - It is the ratio of maximum static friction to the normal force $N$. $f_{s f}=M S F / N$.

Coefficient of Kinetic Friction $\left(\mathrm{f}_{\mathrm{Kf}}\right)$ - It is the ratio of kinetic friction force to the normal force $N$. $f_{k f}=K F / N$.

Cutting - A machining process in which a layer of material is separated from the surface of a workpiece.

Chip - A fragment of material removed from a surface during a machining process.

Buffing - The smoothing of the metal surface and the removal of spikes (peaks and asperities) right after the surface texturing step.

Burnish - To polish a disk surface so as to remove dust particles or minimize asperities on the disk surface.

Plowing - A machining process in which a groove is produced in a surface but material is not separated. Material is displaced plastically ahead of, and to the side of , the machining tool.

Adhesives - The polymer resins commonly used for fastening the abrasive grains to the surface of the abrasive tape. 